Tôhoku Math. Journ.

30 (1978), 277-305.

\title{
ON ONE-PARAMETER GROUPS OF AUTOMORPHISMS, AND EXTENSIONS OF SYMMETRIC OPERATORS ASSOCIATED WITH UNBOUNDED DERIVATIONS IN OPERATOR ALGEBRAS
}

\author{
PALLE E. T. JøRGENSEN*
}

(Received December 18, 1976)

1. Introduction. In quantum field theory and in quantum statistical mechanics the multiple time Green's function $[1,16,17,18,19,24]$ has been studied from different points of view. In [24] it is shown that the vacuum expectation values of a neutral scalar field, interacting with itself, gives rise to a dynamics of a large algebra generated by an abstract "time evolution" together with the time zero field algebra. In [19] the same algebraic considerations are used in describing the dynamics and the Gibbs states of quantum lattice systems with "bad" potentials, i.e., potentials where the time development cannot be described with a oneparameter group of *automorphisms of the given $C^{*}$-algebra of time zero observables. The two approaches, summarized above, have been extended in a recent paper [7] by Bratteli and Robinson.

Common to the earlier works is that the attention has been restricted to Gibbs states and KMS states. In this paper we adapt the algebraic tools of the articles cited above to arbitrary time invariant states. As applications we propose an infinitesimal KMS condition (and ground state condition) for unbounded derivations. Existence theorems are proved for inner limit derivations.

The paper [7] also constructs modular states for derivations (in a special class of nest algebras) which are not generators of one-parameter groups of automorphisms, but the analysis in [7] is based on the onetime Green's function. The conclusions in [7] are therefore of a different kind than the present ones, based on the convergence of the multi-time Green's functions of locally time-invariant states.

Existence theorems for KMS states and ground states have recently been proved in [15] and further analyzed in [10]. Those papers treat the general setting of $C^{*}$-algebras, but assume that the derivation is a generator. Our present results are different from [15] in several respects.

\footnotetext{
* Supported by Odense University, Denmark.
} 
Firstly, they apply to arbitrary inner limit derivations which are not assumed to generate one-parameter groups of automorphisms. This is the essential reason why the multi-time Green's functions are needed. In the case of generators, the states alone suffice.

In the first section we establish a correspondence between dynamics and extensions of certain states on the algebra of time-dependent observables associated with the multi-time Green's function. (Our first theorem is an abstraction of a special case which first appeared in [19].) We have stated these results in the generality of unbounded representations [13], although only bounded representations are needed in the main section. Only little additional work is needed for the extra generality, and applications are pointed out along the way.

Let $\delta$ be an unbounded densely defined *-derivation in a $C^{*}$-algebra $\mathfrak{A}$, and let $\pi_{0}$ be a representation of $\mathfrak{A}$ on a Hilbert space $\mathscr{K}$. Finally, let $H$ be an unbounded self-adjoint operator in $\mathscr{K}$. We say that $H$ extends $\delta$ in the representation $\pi_{0}$ if $\pi_{0}(A) f \in D(H)$ for all $A \in D(\delta)$ and $f \in D(H)$, and

$$
i H \pi_{0}(A) f=\pi_{0}(\delta(A)) f+\pi_{0}(A) i H f .
$$

In the main section of the paper we construct for a general class of derivations $\delta$ a variety of physically relevant representations $\pi_{0}: \mathfrak{A} \rightarrow B(\mathscr{K})$ and self-adjoint operators $H$ which extend $\delta$ in the respective representations $\pi_{0}$. The above mentioned extension theorem is used in combination with the Arzela-Ascoli theorem in constructing the representations $\pi_{0}$.

\section{Extension of states and dynamics.}

Definition 1 . If $\mathfrak{A}$ is a *algebra over the complex numbers with identity $I$, then we denote by $\mathfrak{B}=\mathfrak{B}(\mathfrak{H})$ the free *-algebra generated by pairs of elements $(A, t)$ with $A \in \mathfrak{A}$ and $t \in \boldsymbol{R}$, modulo the relations

$$
\left(A_{1}, t\right)\left(A_{2}, t\right)=\left(A_{1} A_{2}, t\right), \lambda\left(A_{1}, t\right)+\left(A_{2}, t\right)=\left(\lambda A_{1}+A_{2}, t\right),
$$

and $(I, t)=(I, 0)$ for all $t \in R, \lambda \in C$ and $A_{1}, A_{2} \in \mathfrak{A}$. More specifically, let $\mathscr{W}$ denote the ${ }^{*}$-algebra spanned linearly over the complex numbers by strings of elements

$$
P=\left(A_{1}, t_{1}\right)\left(A_{2}, t_{2}\right) \cdots\left(A_{k}, t_{k}\right) \text { with } A_{i} \in \mathfrak{A} \text { and } t_{i} \in R .
$$

Define the *-operation by $P^{*}=\left(A_{k}^{*}, t_{k}\right) \cdots\left(A_{1}^{*}, t_{1}\right)$ with $P$ as above. Let $J$ denote the two sided ${ }^{*}$-ideal generated by the elements

$$
\left(A_{1}, t\right)\left(A_{2}, t\right)-\left(A_{1} A_{2}, t\right), \lambda\left(A_{1}, t\right)+\left(A_{2}, t\right)-\left(\lambda A_{1}+A_{2}, t\right),
$$


and $(I, t)-(I, 0)$. Then $\mathfrak{B}(\mathfrak{A})$ is the quotient *-algebra $\mathscr{W} / J$. Note that $\mathfrak{B}(\mathfrak{A})$ has an identity.

Following [19], [16] and [24], we define a one-parameter group of *-automorphisms $\left\{g_{t}:-\infty<t<\infty\right\}$ of $\mathscr{W}$ by

$$
g_{t}\left(\left(A_{1}, t_{1}\right) \cdots\left(A_{k}, t_{k}\right)\right)=\left(A_{1}, t_{1}+t\right)\left(A_{2}, t_{2}+t\right) \cdots\left(A_{k}, t_{k}+t\right) .
$$

Since $g_{t}$ is a ${ }^{*}$-automorphism of $\mathscr{W}$ for each $t$, the set of elements given by (2) is invariant under $g_{t}$ for all $t$. It follows that the two-sided ideal $J$ is invariant as well. The corresponding one-parameter group of *-automorphisms of the quotient algebra $\mathfrak{B}(\mathfrak{R})=\mathscr{W} / J$ is also denoted by $\left\{g_{t}: t \in \boldsymbol{R}\right\}$.

TheOREM 1. Let $\omega$ be a positive linear functional $\left(\omega\left(P^{*} P\right) \geqq 0\right.$ for all $P \in \mathfrak{B}(\mathfrak{A})$ ) on $\mathfrak{B}(\mathfrak{R})$ satisfying the following conditions

(i ) $\omega\left(g_{t}(P)\right)=\omega(P)$ for all $t \in \boldsymbol{R}$ and $P \in \mathfrak{B}(\mathfrak{A})$

(ii) The mapping $t \rightarrow \omega\left(P^{*} g_{t}(P)\right)$ is continuous on $\boldsymbol{R}$ for all $P \in \mathfrak{B}(\mathfrak{A})$. I. Then there is $a^{*}$-representation (closable in the sense of [13]) $\pi$ of $\mathfrak{B}(\mathfrak{H})$ into possibly unbounded operators with a common dense and invariant domain $\mathscr{D}$ in a Hilbert space $\mathscr{K}$, a cyclic vector $\xi \in \mathscr{D}$, and a strongly continuous unitary representation $U(t)$ of $\boldsymbol{R}$ on $\mathscr{K}$ such that

(a) $\mathscr{D}=\pi(\mathfrak{B}) \xi$

(b) $\omega(P)=(\xi, \pi(P) \xi)$

(c) $U(t) \xi=\xi$

(d) $U(t) \pi(P) U(-t)=\pi\left(g_{t}(P)\right)$ for all $P \in \mathfrak{B}(\mathfrak{R})$ and all $t \in \boldsymbol{R}$.

II. Let $\pi_{0}$ denote the restriction of $\pi$ to $\mathfrak{A}$. Then

$$
\begin{aligned}
& \omega\left(A_{1}, t_{1}\right)\left(A_{2}, t_{2}\right) \cdots\left(A_{k}, t_{k}\right) \\
& \quad=\left(\xi, \pi_{0}\left(A_{1}\right) U\left(t_{2}-t_{1}\right) \pi_{0}\left(A_{2}\right) U\left(t_{3}-t_{2}\right) \cdots U\left(t_{k}-t_{k-1}\right) \pi_{0}\left(A_{k}\right) \xi\right) .
\end{aligned}
$$

The function $\omega\left(\left(A_{1}, t_{1}\right)\left(A_{2}, t_{2}\right)\right)$ is uniformly bounded in $\left(t_{1}, t_{2}\right) \in \boldsymbol{R}^{2}$ for fixed $A_{1}, A_{2} \in \mathfrak{A}$.

III. Moreover, the representation $\pi$ is determined up to unitary equivalence by conditions (a) and (b). The four conditions (a) through (d) determine $\pi$ and $U(t)$ up to unitary equivalence.

PRoof. We will only outline the proof of this version of the GNS construction, omitting many details, since the ingredients are essentially contained in [13], [19] and [21].

I. Let $\omega$ be a state on $\mathfrak{B}$ satisfying (i) and (ii). Let $\mathscr{L}$ denote the left ideal of element $P \in \mathfrak{B}$ such that $\omega\left(P^{*} P\right)=0$. For arbitrary elements $P, Q \in \mathfrak{B}$ we have by the generalized Schwarz's inequality

$$
\omega\left((P Q)^{*} P Q\right)=\omega\left(Q^{*} P^{*} P Q\right) \leqq \omega\left(Q^{*} Q\right)^{1 / 2} \omega\left(Q^{*}\left(P^{*} P\right)^{2} Q\right)^{1 / 2}
$$


We denote by $\mathscr{D}$ the quotient space $\mathfrak{B} / \mathscr{L}$ with inner product

$$
(P+\mathscr{L}, Q+\mathscr{L})=\omega\left(P^{*} Q\right)
$$

It follows from (4) that the inner product is well defined. Similarly $\pi(P)(Q+\mathscr{L})=P Q+\mathscr{L}$ is well defined for all points $[Q]=Q+\mathscr{L}$ in $\mathscr{D}$. If $\xi$ denotes the vector $[I]=[(I, 0)]=[(I, t)]$ then $\mathscr{D}=\pi(\mathfrak{B}) \xi$. We let $\mathscr{K}$ denote the completion of $\mathscr{D}$ with respect to the inner product given by (5) and contend that $\pi$ is a *-representation which satisfies (b). $\mathscr{D}$ is clearly a common dense and invariant domain for this representation. We now define $U(t)$ for $t \in \boldsymbol{R}$ by

$$
U(t) \pi(P) \xi=\pi\left(g_{t}(P)\right) \xi,
$$

and leave to the reader to check that (6) is a good definition; $\|U(t) \pi(P) \xi\|^{2}=$ $\|\pi(P) \xi\|^{2}=\omega\left(P^{*} P\right)$ for all $t$; and $\lim \{\|U(t) \pi(P) \xi-\pi(P) \xi\|: t \rightarrow 0\}=0$.

Here the property (i) and (ii) of the positive linear functional $\omega$ are used. Finally properties (c) and (d) follow from property (i) and (6) combined. II. Let $\pi_{0}$ denote the restriction of $\pi$ to $\mathfrak{A} \approx(\mathfrak{A}, 0)$. Using (c) and (d), we then have, starting with the left hand side of (3)

$$
\begin{aligned}
& \omega\left(\left(A_{1}, t_{1}\right) \cdots\left(A_{k}, t_{k}\right)\right)=\left(\xi, \pi\left(\left(A_{1}, t_{1}\right) \cdots\left(A_{k}, t_{k}\right)\right) \xi\right) \\
& \quad=\left(\xi, \pi\left(g_{t_{1}}\left(A_{1}\right)\right) \cdots \pi\left(g_{t_{k}}\left(A_{k}\right)\right) \xi\right) \\
& \quad=\left(\xi, U\left(t_{1}\right) \pi_{0}\left(A_{1}\right) U\left(-t_{1}\right) U\left(t_{2}\right) \pi_{0}\left(A_{2}\right) U\left(-t_{2}\right) \cdots \pi_{0}\left(A_{k}\right) U\left(-t_{2}\right) \xi\right) \\
& \quad=\left(\xi, \pi_{0}\left(A_{1}\right) U\left(t_{2}-t_{1}\right) \pi_{0}\left(A_{2}\right) \cdots U\left(t_{k}-t_{k-1}\right) \pi_{0}\left(A_{k}\right) \xi\right),
\end{aligned}
$$

and (3) follows. Uniform boundedness of $\omega\left(\left(A_{1}, t_{1}\right)\left(A_{2}, t_{2}\right)\right)$ in $\left(t_{1}, t_{2}\right)$ follows from the estimate

$$
\left|\left(\xi, \pi_{0}\left(A_{1}\right) U\left(t_{2}-t_{1}\right) \pi_{0}\left(A_{2}\right) \xi\right)\right| \leqq\left\|\pi_{0}\left(A_{1}^{*}\right) \xi\right\|\left\|\pi_{0}\left(A_{2}\right) \xi\right\| .
$$

III. The uniqueness of $\pi$ and $U(t)$ follows as in [13] and [21].

Our next result concerns extensions of positive linear functionals on $\mathfrak{A}$. (A positive linear functional will be called a state in the sequel even if it is not normalized). We know that states on the subalgebra $\mathfrak{A}$ of $\mathfrak{B}(\mathfrak{A})$ always extend to states of $\mathfrak{A}(\mathfrak{B})$. See for example [20]. But the extension may in general be highly non-unique. However, we have

THEOREM 2. Let $\mathfrak{A}$ be $a^{*}$-algebra over the complex numbers, and let $\pi_{0}$ be $a{ }^{*}$-representation of $\mathfrak{A}$ with a common dense and invariant domain $\mathscr{D}$ in a Hilbert space $\mathscr{K}$. Let $\xi$ be a vector in $\mathscr{D}$ and let $\omega_{0}$ be the vector state on $\mathfrak{A}$ given by $\omega_{0}(A)=\left(\xi, \pi_{0}(A) \xi\right)$ for $A \in \mathfrak{A}$.

If $U(t)$ is a strongly continuous unitary one-parameter group on $\mathscr{\varkappa}$ satisfying

(e ) $U(t) \mathscr{D}=\mathscr{D}$, and 
(f) $U(t) \xi=\xi$ for all $t \in \boldsymbol{R}$,

then the linear extension $\omega$ given by (3) to $\mathfrak{B}(\mathfrak{A})$ of $\omega_{0}$ is positive, i.e., $\omega\left(P^{*} P\right) \geqq 0$ for all $P \in \mathfrak{B}(\mathfrak{A})$, and satisfies conditions (i) and (ii) of Theorem 1.

Proof. Note that since $\xi \in \mathscr{D}, \pi_{0}(\mathfrak{A}) \mathscr{D} \subset \mathscr{D}$, and (e) hold, we may define $\omega\left(\left(A_{1}, t_{1}\right) \cdots\left(A_{k}, t_{k}\right)\right)$ by the right hand side of (3) for every product of the form $\left(A_{1}, t_{1}\right) \cdots\left(A_{k}, t_{k}\right)$ in $\mathfrak{B}(\mathfrak{A})$. Since $\mathfrak{B}(\mathfrak{R})$ is spanned as a linear space by such products, the linear extension of $\omega_{0}$ to $\mathfrak{B}(\mathfrak{A})$ is uniquely given this way, and it is clear that $\omega$ extends $\omega_{0}$.

Using Definition 1 we see that for every $P \in \mathfrak{B}(\mathfrak{R})$ there is a positive integer $k$ such that $P=\sum P_{n}$, where the elements $P_{n}$ are all of the form (1) and where $n$ runs over a finite index set, also depending on $P$. (To see this, we may insert the identity element appropriately and use the multi linearity built into Definition 1.) We must show that

$$
\omega\left(P^{*} P\right)=\sum_{n, m} \omega\left(P_{n}^{*} P_{m}\right) \geqq 0
$$

where $\omega\left(P_{n}^{*} P_{m}\right)$ is defined by (3) for each pair of indices $n$ and $m$. In order to avoid too heavy index notation, we restrict ourself to the case $k=2$ leaving the analogues cases $k>2$ to the reader. Thus we assume that for each index $n$ there are pairs $A_{n}, B_{n} \in \mathfrak{A}$ and $s_{n}, t_{n} \in \boldsymbol{R}$ such that $P_{n}=\left(A_{n}, t_{n}\right)\left(B_{n}, s_{n}\right)$. Then

$$
\omega\left(P_{n}^{*} P_{m}\right)=\left(\pi_{0}\left(A_{n}\right) U\left(s_{n}-t_{n}\right) \pi_{0}\left(B_{n}\right) \xi, \pi_{0}\left(A_{m}\right) U\left(s_{m}-t_{m}\right) \pi_{0}\left(B_{m}\right) \xi\right)
$$

If we define vectors $\eta_{n}$ and $\eta$ by

$$
\eta_{n}=\pi_{0}\left(A_{n}\right) U\left(s_{n}-t_{n}\right) \pi_{0}\left(B_{n}\right) \xi \text { and } \eta=\sum_{n} \eta_{n},
$$

it follows that $\omega\left(P^{*} P\right)=\sum_{n, m}\left(\eta_{n}, \eta_{m}\right)=\left(\sum_{n} \eta_{n}, \sum_{m} \eta_{m}\right)=(\eta, \eta)=\|\eta\|^{2} \geqq 0$ showing that $\omega$ is a positive linear functional.

It is clear from (3) that $\omega$ extends $\omega_{0}$. We must finally verify conditions (i) and (ii).

By linearity it is enough to verify these conditions for elements $P$ of the form (1). For such elements condition (i) is clear because $\omega(P)$ depends only on the differences $\left(t_{2}-t_{1}, \cdots, t_{k}-t_{k-1}\right)$, and the $k$ vectors $\left(t_{1}, \cdots, t_{k}\right)$ and $\left(t_{1}+t, \cdots, t_{k}+t\right)$ have the same difference-vector. Finally condition (ii) follows immediately from the strong continuity of $U(t)$.

REMARK 1. For many interesting states $\omega$ on $\mathfrak{B}(\mathfrak{R})$, the corresponding representation $\pi$ described in Theorem 1 is bounded, i.e., $\pi(P)$ is bounded on $\mathscr{K}$ for all $P \in \mathfrak{B}(\mathfrak{H})$. This is the case when $U(t)$ in (3) 
implements an automorphism group of $\mathfrak{A}$ and for weak $*$ limits of such states $\omega$. These states satisfy the following conditon

$$
\omega\left(P^{*}\left(A^{*} A, t\right) P\right) \leqq\|A\|^{2} \omega\left(P^{*} P\right)
$$

for $P \in \mathfrak{B}(\mathfrak{U}), A \in \mathfrak{A}$, and $t \in \boldsymbol{R}$, which in turn implies boundedness of $\pi((A, t))$, and hence of $\pi(Q)$ for all $Q \in \mathfrak{B}(\mathfrak{U})$.

As a corollary to this remark we have

CoRollary 2. Suppose the representation $\pi_{0}$ of Theorem 2 is a bounded representation of a $C^{*}$-algebra $\mathfrak{A}$ on the Hilbert space $\mathscr{K}$. Then the representation $\pi$ induced by the extended state $\omega$ is also bounded on $\mathscr{K}$, i.e., $\pi(Q)$ bounded for all $Q \in \mathfrak{B}(\mathfrak{U})$.

Proof. We need only to verify the estimate (8) for the extended state $\omega$. With $P$ given by (1) we have

$$
\begin{aligned}
\omega\left(P^{*}\left(A^{*} A, t\right) P\right) & =\omega((A, t) P)^{*}((A, t) P) \\
& =\left\|\pi_{0}(A) U\left(t_{1}-t\right) \pi_{0}\left(A_{1}\right) U\left(t_{2}-t_{1}\right) \cdots U\left(t_{k}-t_{k-1}\right) \pi_{0}\left(A_{k}\right) \xi\right\|^{2} \\
& \leqq\left\|\pi_{0}(A)\right\|^{2}\left\|\pi_{0}\left(A_{1}\right) U\left(t_{2}-t_{1}\right) \cdots U\left(t_{k}-t_{k-1}\right) \pi_{0}\left(A_{k}\right) \xi\right\|^{2} \\
& \leqq\|A\|^{2} \omega\left(P^{*} P\right) \quad \text { q.e.d. }
\end{aligned}
$$

\section{KMS states on $\mathfrak{B}(\mathfrak{U})$.}

Definition 2. Let $\mathfrak{A}$ be a *-algebra over $\boldsymbol{C}$, and let $\pi_{0}$ be a ${ }^{*}$-representation of $\mathfrak{A}$ on a common dense and invariant domain $\mathscr{D}$ in a Hilbert space $\mathscr{K}$. Let $U(t)$ be a strongly continuous representation of $\boldsymbol{R}$ satisfying conditions (e) and (f) for some vector $\xi \in \mathscr{D}$. We then say that the state $\omega_{0}(A)=\left(\xi, \pi_{0}(A) \xi\right)$ is KMS at inverse temperature $\beta>0$ for $U(t)$ if one of the following two equivalent conditions hold

$$
\int_{-\infty}^{\infty} \varphi(t)\left(\xi, \pi_{0}(A) U(t) \pi_{0}(B) \xi\right) d t=\int_{-\infty}^{\infty} \varphi(t+i \beta)\left(\xi, \pi_{0}(B) U(-t) \pi_{0}(A) \xi\right) d t
$$

for every entire analytic function $\varphi$ such that

$$
\hat{\varphi}(x)=\int e^{-i t x} \varphi(t) d t
$$

belongs to the space $\mathscr{D}(\boldsymbol{R})$ of compactly supported $C^{\infty}$-functions on the line. ( $\mathrm{h}$ ) There is a bounded continuous function $F(z)$ on

$$
\{z: 0 \leqq \operatorname{Im} z \leqq \beta\}
$$

which is analytic in the interior of this strip and satisfies

$$
F(t)=\left(\xi, \pi_{0}(A) U(t) \pi_{0}(B) \xi\right) \quad \text { and } \quad F(t+i \beta)=\left(\xi, \pi_{0}(B) U(-t) \pi_{0}(A) \xi\right)
$$

for $t \in \boldsymbol{R}$.

Naturally there are similar but quite symmetric conditions for $\beta<0$. 
The equivalence of $(\mathrm{g})$ and $(\mathrm{h})$ is a consequence of the following lemma which is implicit in [2].

LEMMA 1. Let $f_{0}$ and $f_{\beta}$ be bounded continuous functions on $\boldsymbol{R}$, which are both Fourier transforms of finite complex measures on $\boldsymbol{R}$. Then the following conditions are equivalent.

$\left(\mathrm{g}^{\prime}\right) \quad \int_{-\infty}^{\infty} \varphi(t) f_{0}(t) d t=\int_{-\infty}^{\infty} \varphi(t+i \beta) f_{\beta}(t) d t$ for every $\varphi$ as in $(\mathrm{g})$, and

$\left(\mathrm{h}^{\prime}\right)$ There is a bounded analytic function $F$ as in $(\mathrm{h})$ such that $F(t)=$ $f_{0}(t)$ and $F(t+i \beta)=f_{\beta}(t)$ for all $t \in \boldsymbol{R}$.

Note that the lemma does indeed apply. The function $f_{0}(t)=$ $\left(\xi, \pi_{0}(A) U(t) \pi_{0}(B) \xi\right)$ is bounded (by $\left.\left\|\pi_{0}\left(A^{*}\right) \xi\right\|\left\|\pi_{0}(B) \xi\right\|\right)$ and continuous on $\boldsymbol{R}$. Let $U(t)=\int e^{i t x} E(d x)$ be the spectral decomposition of $U(t)$, and put $\mu_{0}(d x)=\left(\pi_{0}\left(A^{*}\right) \xi, E(d x) \pi_{0}(B) \xi\right)$. Then $\left\|\mu_{0}\right\|<\infty$ and $f_{0}=\hat{\mu}_{0}$.

The significance of Definition 2 is due to the fact that the states in question are modular states. Starting with a state $\omega_{0}(A)=\left(\xi, \pi_{0}(A) \xi\right)$ for some representation $\pi_{0}$ of $\mathfrak{A}$ on a Hilbert space $\mathscr{K}$, we define the cyclic subspace $\mathscr{H}$ as the closed linear subspace spanned by $\pi_{0}(\mathfrak{U}) \xi$ and denote by $\pi_{1}$ the restriction of $\pi_{0}$ to $\mathscr{\mathscr { C }}$.

Proposition 1. Suppose that $\pi_{1}$ is a bounded representation on $\mathscr{K}$, and $\omega_{0}$ is a KMS state for some $\beta>0$. Then $\xi$ is a cyclic and separating vector for the von Neumann algebra $\pi_{1}(\mathfrak{A})^{\prime \prime}$, i.e., the bi-commutant of $\pi_{1}(\mathfrak{A})$ in $B(\mathscr{H})$.

Proof. It is clear that $\xi$ is cyclic for $\pi_{1}(\mathfrak{A})^{\prime \prime}$. Let $X \in \pi_{1}(\mathfrak{A})^{\prime \prime}$ be such that $X^{*} \xi=0$. By Kaplansky's density theorem there is a bounded sequence $B_{n} \in \mathfrak{A}$ such that $\pi_{1}\left(B_{n}\right) \xi \rightarrow X \xi$ and $\pi_{1}\left(B_{n}^{*}\right) \xi \rightarrow 0$. We conclude from (g) that $\int \varphi(t)\left(\pi_{0}(A) \xi, U(t) X \xi\right) d t=0$ for all $\varphi$ with $\hat{\varphi} \in \mathscr{D}$ and all $A \in \mathfrak{A}$. This in turn implies $X \xi=0$. For arbitrary $Y \in \pi_{1}(\mathfrak{A})^{\prime \prime}$ we then have $(X Y)^{*} \xi=Y^{*} X^{*} \xi=0$, and by the above $X Y \xi=0$. Hence, $X$ is identically zero, since this holds for all $Y$. q.e.d.

The conditions of Definition 2 are formulated in such a way that they are easy to check in applications. However, the dynamics is described by a one-parameter group of *automorphisms $g_{t}$ and a state $\omega$ on $\mathfrak{B}(\mathfrak{U})$. Therefore one is really interested in knowing the KMS property for the data $\left(\mathfrak{B}(\mathfrak{U}), g_{t}, \omega\right)$.

DeFinition 3. We say that a state $\omega$ on $\mathfrak{B}(\mathfrak{A})$ is a KMS state for inverse temperature $\beta>0$ if condition $\left(g^{\prime}\right)$ (or equivalently $\left(h^{\prime}\right)$ ) is satisfied for the functions 


$$
f_{0}(t)=\omega\left(P g_{t}(Q)\right) \text { and } f_{\beta}(t)=\omega\left(g_{t}(Q) P\right)
$$

for every pair of elements $P$ and $Q$ in $\mathfrak{B}(\mathfrak{A})$.

The following theorem is therefore of principal importance.

THEOREM 3. Let the data $\left(\pi_{0}, \mathfrak{A}, \mathscr{K}, \xi, U(t)\right)$ be as above and as in Theorem 2. Let $\omega_{0}$ be the vector state given by $\omega_{0}(A)=\left(\xi, \pi_{0}(A) \xi\right), A \in \mathfrak{A}$, and let $\omega$ be the extension given by (3) to $\mathfrak{B}(\mathfrak{X})$ of $\omega_{0}$.

Then $\omega$ is KMS for inverse temperature $\beta$ if and only if for every positive integer $k$ and every set $A_{1}, \cdots, A_{k}$ of elements in $\mathfrak{A}$ there exists a holomorphic $F\left(z_{1}, \cdots, z_{k}\right)$ in the region $0<\operatorname{Im} z_{1}<\operatorname{Im} z_{2}<\cdots<$ $\operatorname{Im} z_{k}<\beta$, continuous up to the boundary, such that the boundary value of $F$ for $\operatorname{Im} z_{1}=\cdots=\operatorname{Im} z_{j}=0$ and $\operatorname{Im} z_{j+1}=\cdots=\operatorname{Im} z_{k}=\beta$, i.e., $F\left(t_{1}, \cdots, t_{j}, t_{j+1}+i \beta, \cdots, t_{k}+i \beta\right)$, is the function

$$
\begin{aligned}
& \left(\xi, \pi_{0}\left(A_{j+1}\right) U\left(t_{j+2}-t_{j+1}\right) \cdots U\left(t_{k}-t_{k-1}\right) \pi_{0}\left(A_{k}\right)\right. \\
& \left.U\left(t_{1}-t_{k}\right) \pi_{0}\left(A_{1}\right) \cdots U\left(t_{j}-t_{j-1}\right) \pi_{0}\left(A_{j}\right) \xi\right) .
\end{aligned}
$$

Proof. The proof depends in a crucial way on the main result of [2], Theorem 3.1, or rather the proof of this result. It is pointed out in [2, Remark 3.2] that [2, Theorem 3.1] and its proof are stated in a form which includes the applications to Wightman fields. By the same reasoning it follows that [2] applies to the present case with unbounded representations $\pi_{0}$.

Suppose first that $\omega$ is KMS for some $\beta>0$. By [2, Theorem 3.1] we see that there is a holomorphic function $F$ in $0<\operatorname{Im} z_{1}<\cdots<$ $\operatorname{Im} z_{k}<\beta$, continuous up to the boundary, such that for all $j=1, \cdots, k$

$$
\begin{aligned}
F( & \left.t_{1}, \cdots, t_{j}, t_{j+1}+i \beta, \cdots, t_{k}+i \beta\right) \\
\quad & =\omega\left(g_{t_{j+1}}\left(A_{j+1}\right) \cdots g_{t_{k}}\left(A_{k}\right) g_{t_{1}}\left(A_{1}\right) \cdots g_{t_{j}}\left(A_{j}\right)\right) \\
& =\omega\left(\left(A_{j+1}, t_{j+1}\right) \cdots\left(A_{k}, t_{k}\right)\left(A_{1}, t_{1}\right) \cdots\left(A_{j}, t_{j}\right)\right) \\
& =\left(\xi, \pi_{0}\left(A_{j+1}\right) U\left(t_{j+2}-t_{j+1}\right) \cdots \pi_{0}\left(A_{k}\right) U\left(t_{1}-t_{k}\right) \pi_{0}\left(A_{1}\right) \cdots U\left(t_{j}-t_{j-1}\right) \pi_{0}\left(A_{j}\right) \xi\right) .
\end{aligned}
$$

Conversely, suppose that for every $k$-tuple of elements in $\mathfrak{A}$ there exists a holomorphic function with the stated boundary values. We must show that for every pair of elements $P, Q \in \mathfrak{B}(\mathfrak{U})$ there exists a bounded holomorphic function $\Phi(z)$ say in $0<\operatorname{Im} z<\beta$, continuous up to the boundary, which satisfies

$$
\Phi(t)=\omega\left(P g_{t}(Q)\right) \text { and } \Phi(t+i \beta)=\omega\left(g_{t}(Q) P\right) .
$$

By linearity it is enough to consider elements $P$ and $Q$ in $\mathfrak{B}(\mathfrak{A})$ of the form $P=\left(A_{1}, s_{1}\right) \cdots\left(A_{m}, s_{m}\right)$ and $Q=\left(B_{1}, t_{1}\right) \cdots\left(B_{n}, t_{n}\right)$. Corresponding to the $(k=m+n)$ tuple of elements $\left(A_{1}, \cdots, A_{m}, B_{1}, \cdots, B_{n}\right)$ there exists 
a holomorphic function $F\left(z_{1}, \cdots, z_{m+n}\right)$ in $0<\operatorname{Im} z_{1}<\cdots<\operatorname{Im} z_{m+n}<\beta$ with the boundary values stated in the theorem. In particular

$$
\begin{aligned}
& F\left(s_{1}, \cdots, s_{m}, t_{1}+t+i \beta, \cdots, t_{n}+t+i \beta\right) \\
& \quad=\left(\xi, \pi_{0}\left(B_{1}\right) U\left(t_{2}-t_{1}\right) \cdots \pi_{0}\left(B_{n}\right) U\left(s_{1}-t_{n}-t\right) \pi_{0}\left(A_{1}\right) U\left(s_{2}-s_{1}\right) \cdots\right. \\
& \left.\quad U\left(s_{m}-s_{m-1}\right) \pi_{0}\left(A_{m}\right) \xi\right) \\
& \quad=\omega\left(\left(B_{1}, t_{1}+t\right) \cdots\left(B_{n}, t_{n}+t\right)\left(A_{1}, s_{1}\right) \cdots\left(A_{m}, s_{m}\right)\right) .
\end{aligned}
$$

Similarly

$$
\begin{aligned}
& F\left(s_{1}, \cdots, s_{m}, t_{1}+t, \cdots, t_{n}+t\right) \\
& \quad=\omega\left(\left(A_{1}, s_{1}\right) \cdots\left(A_{m}, s_{m}\right)\left(B_{1}, t_{1}+t\right) \cdots\left(B_{n}, t_{n}+t\right)\right) .
\end{aligned}
$$

For $t \in \boldsymbol{R}$ and $0 \leqq \alpha \leqq \beta$ we now define

$$
\Phi(\mathrm{t}+i \alpha)=F\left(s_{1}, \cdots, s_{m}, t_{1}+t+i \alpha, \cdots, t_{n}+t+i \alpha\right) .
$$

Since $F$ is analytic as a function of $m+n$ complex variables $0<$ $\operatorname{Im} z_{1}<\cdots<\operatorname{Im} z_{m+n}<\beta$ and continuous up to the boundary, we conclude that $\Phi(z)$ is analytic in the complex variable $z=t+i \alpha, 0<\alpha<\beta$, and continuous up to the boundary. We recall that the numbers $s_{1}, \cdots, s_{m}$, $t_{1}, \cdots, t_{n}$, depending only on $P$ and $Q$, are fixed. It follows from (10) and (11) that $\Phi(z)$ satisfies the right boundary conditions (9).

To conclude the proof we only need to show that $\Phi(t+i \alpha)$ is uniformly bounded in the strip $0 \leqq \alpha \leqq \beta$. By the three-line lemma of Doetsch, it is enough to show that $\sup \{|\Phi(t)|: t \in \boldsymbol{R}\}$ and $\sup \{|\Phi(t+i \beta)|$ : $t \in \boldsymbol{R}\}$ are finite. But it follows from (11) and (10) that the first number is dominated by

$$
\left\|\pi_{0}\left(A_{m}^{*}\right) U\left(s_{m-1}-s_{m}\right) \cdots \pi_{0}\left(A_{1}^{*}\right) \xi\right\|\left\|\pi_{0}\left(B_{1}\right) U\left(t_{2}-t_{1}\right) \cdots \pi_{0}\left(B_{n}\right) \xi\right\|,
$$

and the second by

$$
\left\|\pi_{0}\left(B_{n}^{*}\right) U\left(t_{n-1}-t_{n}\right) \cdots \pi_{0}\left(B_{1}^{*}\right) \xi\right\|\left\|\pi_{0}\left(A_{1}\right) U\left(s_{2}-s_{1}\right) \cdots \pi_{0}\left(A_{m}\right) \xi\right\| .
$$

q.e.d.

4. Existence of KMS states and ground states for $C^{*}$-systems. We shall show in this section that if $\mathfrak{A}$ is a $C^{*}$-algebra with unit and with at least one-trace state, then there is associated to each inner limit derivation in $\mathfrak{A}$, and each non-zero inverse temperature, a dynamical system which is in "equilibrium" KMS state. This result is a natural generalization of the result [15, Theorem 3.2] which states that every approximately inner one-parameter group of ${ }^{*}$-automorphisms of a $C^{*}$ algebra as above has KMS states at every non-zero inverse temperature. The formulation of our result at the same time proposes an infinitesimal 
definition of KMS states. We give a similar discussion of ground states for an inner limit derivation $\delta$ in an arbitrary $C^{*}$-algebra.

The section concludes with a discussion of extensions of $\delta$ in the representations induced by the above mentioned states. In the case of ground states, we compare our extension with the Friedrichs extension, and with the extension obtained in classical dilation theory.

Definition 4. Following [5] we say that an unbounded *-derivation $\delta$ in a $C^{*}$-algebra $\mathfrak{A}$ is an inner limit derivation if there is a core $\mathfrak{A}_{0}$ for $\delta$ and a sequence $\delta_{n}$ of inner ${ }^{*}$-derivations on $\mathfrak{U}$ such that $\delta(A)=\lim _{n} \delta_{n}(A)$ for all $A \in \mathfrak{A}_{0}$ Specifically, the requirement on $\mathfrak{A}_{0}$ is that for every element $A$ in the domain of $\delta$ there is a sequence $A_{n}$ of elements in $\mathfrak{A}_{0}$ such that $A_{n} \rightarrow A$ and $\delta\left(A_{n}\right) \rightarrow \delta(A)$.

Note that normal derivations [14] are a special class of inner limit derivations. Also note that, since bounded derivations are inner if $\mathfrak{A}$ is simple [20], it is enough to assume that the approximating derivations $\delta_{n}$ are bounded if $\mathfrak{A}$ is simple.

An inner limit derivation is said to be regular if it is possible to choose the core $\mathfrak{A}_{0}$ and the approximating sequence $\left\{\delta_{n}\right\}$ in such a way that $\sup \left\|\delta_{n}^{2}(A)\right\|$ is finite for all $A \in \mathfrak{A}_{0}$. It is known, [14], that every normal derivation of finite type is a regular inner limit derivation.

Notation. Suppose $\delta_{n}(A)=\left[i H_{n}, A\right]$ for a sequence of elements $H_{n}=H_{n}^{*} \in \mathfrak{A}$, then we put $\alpha_{n}(t)(A)=e^{i t H_{n}} A e^{-i t H_{n}}$.

LEMMA 2. Let $\delta$ be an inner limit derivation in a $C^{*}$-algebra $\mathfrak{A}$ with unit. Let $\mathfrak{H}_{0}$ be a core for $\delta$, and let $\left\{H_{n}\right\}$ be a sequence of hermitian elements in $\mathfrak{A}$ such that $\delta(A)=\lim _{n}\left[i H_{n}, A\right]$ for all $A \in \mathfrak{A}_{0}$. Let $\left\{\omega_{n}\right\}$ be a corresponding sequence of states on $\mathfrak{A}$ such that

$$
\omega_{n}\left(e^{i t H_{n}} A e^{-i t H_{n}}\right)=\omega_{n}(A) \text { for all } t \in \boldsymbol{R} \text { and } A \in \mathfrak{X} \text {. }
$$

Then there exists a state $\tilde{\omega}$ on $\mathfrak{B}(\mathfrak{A})$, such that for every k-tuple of elements $A_{1}, \cdots, A_{k}$ in $\mathfrak{A}$ there is a subsequence $\left\{n_{p}\right\}$ of the integers satisfying

$$
\tilde{\omega}\left(A_{1}, t_{1}\right) \cdots\left(A_{k}, t_{k}\right)=\lim _{n_{p}} \omega_{n_{p}}\left(\alpha_{n_{p}}\left(t_{1}\right)\left(A_{1}\right) \cdots \alpha_{n_{p}}\left(t_{k}\right)\left(A_{k}\right)\right),
$$

the convergence being uniform for $\left(t_{1}, \cdots, t_{k}\right)$ in compact subsets of $\boldsymbol{R}^{k}$.

The state $\tilde{\omega}$ satisfies conditions (i) and (ii) of Theorem 1 and (8) of Remark 3(b).

We conclude from Theorem 1 and Remark $3(\mathrm{~b})$ that there is a bounded representation $\pi$ of $\mathfrak{B}(\mathfrak{X})$ on a Hilbert space $\mathscr{K}$, a vector $\xi \in \mathscr{K}$, and a 
continuous unitary representation $U(t)$ on $\mathscr{K}$ such that $\tilde{\omega}(P)=(\xi, \pi(P) \xi)$, $U(t) \xi=\xi$, and $U(t) \pi(P) U(-t)=\pi\left(g_{t} P\right)$ for all $P \in \mathfrak{B}(\mathfrak{U})$ and $t \in \boldsymbol{R}$.

LEMMA 3. Let $\delta$ be an inner limit derivation which is also regular and let $\omega_{n}$ be a sequence of $\alpha_{n}(t)$-invariant states as in Lemma 2. Let $H$ be the infinitesimal generator of $U(t)$, and let $\pi_{0}$ be the restriction of $\pi$ to $\mathfrak{A}$.

If $D(\delta)$ denotes the domain of $\delta$, then $\pi_{0}(D(\delta)) \xi$ is contained in the domain of $H$ and

$$
i H\left(\pi_{0}(A) \xi\right)=\pi_{0}(\delta(A)) \xi \text { for all } A \in D(\delta) .
$$

Before starting the proof, we comment on the conclusion (14) and, point out two consequences.

As mentioned in the Introduction, we are interested in the cases where the algebra of time zero observables $\mathfrak{A}$ is not invariant under the dynamics. We say that $\mathfrak{A}$ is invariant under the dynamics if for every $A \in \mathfrak{A}$ the sequence $\alpha_{n}(t)(A)$ is convergent in $\mathfrak{A}$, and if $\alpha(t)(A)=\lim _{n} \alpha_{n}(t)(A)$ defines a strongly continuous one-parameter group of ${ }^{*}$-automorphisms on $\mathfrak{A}$.

Using the considerations above, we may regard $\pi_{0}(\mathfrak{U})$ as an algebra of operators on $\mathscr{H}$. We denote by $M$ the bi-commutant of $\pi_{0}(\mathfrak{U})$ in $B(\mathscr{H})$, i.e., $M=\pi_{0}(\mathfrak{A})^{\prime \prime}$. The invariance conditions

$$
\begin{aligned}
& U(t) M U(-t)=M \quad \text { for all } t \in \boldsymbol{R}, \\
& U(t) \mathscr{H}=\mathscr{H} \text { for all } t \in \boldsymbol{R},
\end{aligned}
$$

are of interest. It is clear that (15) implies (16), and that both are satisfied when $\mathfrak{A}$ is invariant.

ObSeRvation 1. Suppose there is a subset $S$ of $D_{\infty}(\delta)$ (i.e., elements in $\mathfrak{A}$ which belong to the domain of each of the operators $\left.\delta, \delta^{2}, \cdots, \delta^{k}, \cdots\right)$ such that $\pi_{0}(S) \xi$ is dense in $\mathscr{H}$, and such that the vectors in $\pi_{0}(S) \xi$ are quasi-analytic $[6,11]$ for $H$. Then (16) is satisfied.

Proof. It is enough to show that if $A \in S$ and $f \in \mathscr{H}^{\perp}=\mathscr{K} \ominus \mathscr{H}$, then $\left(f, U(t) \pi_{0}(A) \xi\right)_{\mathscr{C}}=0$ for all $t$. But in view of (14) in Lemma 3 above, we have

$$
\left.\left(\frac{d}{d t}\right)^{k}\left(f, U(t) \pi_{0}(A) \xi\right)_{\mathscr{K}}\right|_{t=0}=\left(f,(i H)^{k} \pi_{0}(A) \xi\right)_{\mathscr{K}}=\left(f, \pi_{0}\left(\delta^{k}(A)\right) \xi\right)_{\mathscr{K}}=0 .
$$

The conclusion follows from the Denjoy-Carleman theorem, since $\pi_{0}(A) \xi$ is quasi-analytic for $H$.

More generally we have 
Proposition 2. Let $H_{0}$ denote the symmetric operator in $\mathscr{K}$ which is given on $\pi_{0}(D(\delta)) \xi$ by

$$
i H_{0}\left(\pi_{0}(A) \xi\right)=\pi_{0}(\delta(A)) \xi=i H\left(\pi_{0}(A) \xi\right) .
$$

Suppose $H_{0}$ is essentially self-adjoint. (1) Then (16) is satisfied. (2) If $\tilde{\omega}$ is also a KMS state, then (15) is satisfied as well, and $U(t)$ coincides with the modular group $\Delta^{i t}$ of the cyclic and separating vector $\xi$.

Proof. Since $H_{0}$ is essentially self-adjoint, the closure $H_{0}$ defines a unitary representation $U_{0}(t)=e^{i t H_{0}}$ on $\mathscr{K}$. Let $f$ be an arbitrary vector in $D\left(\bar{H}_{0}\right)$ and let $\left\{A_{n}\right\}$ be a sequence in $D(\delta)$ such that $\pi_{0}\left(A_{n}\right) \xi \rightarrow f$, and $H_{0}\left(\pi_{0}\left(A_{n}\right) \xi\right) \rightarrow H_{0} f$. From (17) we conclude that $\bar{H}_{0} f=H f$. We therefore have

$$
-i \frac{d}{d t} U(t) U_{0}(-t) f=U(t)\left(H-\bar{H}_{0}\right) U_{0}(-t) f \equiv 0,
$$

Since $U_{0}(-t) f \in D\left(\bar{H}_{0}\right)$, and hence $U(t) \equiv U_{0}(t)$. This concludes the proof of part (1). Part (2) follows from [7, Theorem 2].

q.e.d.

The assumptions of Proposition 2 have been verified in applications to Bose gases [8] in a specific model.

We now use the lemmas to construct, for every inner limit derivation $\delta$ and every $\beta>0$, an "equilibrium" (or KMS) dynamical system $\left(\pi_{0}, \mathfrak{A}, \mathscr{K}, \xi, U(t)\right)$ for inverse temperature $\beta$. We say that the system is in "equilibrium" if the state on $\mathfrak{B}(\mathfrak{A})$ determined by the invariant vector $\xi$ is a KMS state in the sense of Definition 3. The infinitesimal generator of $U(t)$ turns out to be an extension of $\delta$ in the representation $\pi$.

THEOREM 4. Let $\delta$ be an inner limit *-derivation in a $C^{*}$-algebra $\mathfrak{A}$ with unit. Suppose $\mathfrak{A}$ has at least one trace state. Then for each $\beta>0$ there exists a dynamical system $\left(\pi_{0}, \mathfrak{A}, \mathscr{K}, \xi, U(t)\right)$ such that $\xi$ defines a KMS state on $\mathfrak{B}(\mathfrak{U})$ at inverse temperature $\beta$.

If $\delta$ is also regular, then the corresponding infinitesimal generator $H=-\left.i(d / d t) U(t)\right|_{t=0}$ satisfies

$$
i H\left(\pi_{0}(A)\right) \xi=\pi_{0}(\delta(A)) \xi
$$

for all $A$ in the domain of $\delta$.

$A$ completely analogous result holds for ground states. Let $\mathfrak{A}$ be a *-algebra. We say that a state $\tilde{\omega}$ on $\mathfrak{B}(\mathfrak{A})$ is a ground state if for every positive integer $k$ and every set of elements $A_{1}, \cdots, A_{k}$ in $\mathfrak{A}$ the multitime Green's function

$$
G\left(A_{1}, \cdots, A_{k} ; t_{1}, \cdots, t_{k}\right)=\tilde{\omega}\left(\left(A_{1}, t_{1}\right) \cdots\left(A_{k}, t_{k}\right)\right)
$$


is the boundary value on $\boldsymbol{R}^{k}$ of a function $F\left(z_{1}, \cdots, z_{k}\right)$ which is analytic in $\operatorname{Im} z_{1}<\operatorname{Im} z_{2}<\cdots<\operatorname{Im} z_{k}$, and continuous and uniformly bounded on the closure of this domain.

TheOREM 5. Let $\delta$ be an inner limit *-derivation in a $C^{*}$-algebra $\mathfrak{A}$ with unit. Then there is a dynamical system $\left(\pi_{0}, \mathfrak{A}, \mathscr{K}, \xi, U(t)\right)$ such that $\xi$ defines a ground state on $\mathfrak{B}(\mathfrak{H})$.

In the event that $\delta$ is also regular, identity (14) holds for the generator $H$ of $U(t)$, and the restriction of $H$ to $\pi_{0}(D(\delta)) \xi$ defines a symmetric operator with non-negative spectrum.

Note that the theorems may be viewed as corollaries to Lemmas 2 and 3. We now pass to the proofs.

PRoofs. Starting with Lemma 2, we recall that $\delta$ is an inner limit derivation with an approximating sequence $\left\{H_{n}\right\}$ of hermitian elements. $\left\{\omega_{n}\right\}$ denotes a corresponding sequence of states such that $\omega_{n}$ is invariant under $\alpha_{n}(t)=e^{i t H_{n}} \cdot e^{-i t H_{n}}$ for all $n$, i.e., $\omega_{n} \alpha_{n}(t)=\omega_{n}$.

By weak-*compactness of the state space of $\mathfrak{A}$, we first note that there exists a subsequence of $\left\{\omega_{n}\right\}$ which is weak-* convergent. We shall denote this subsequence also by $\left\{\omega_{n}\right\}$. We denote the corresponding limit state by $\omega$, i.e.

$$
\omega=\lim _{n} \omega_{n}
$$

Using a simple version of Theorem 2 and Corollary 2 (or by direct verification) we then observe that, for each $n$, the definition

$$
\tilde{\omega}_{n}\left(\left(A_{1}, t_{1}\right) \cdots\left(A_{k}, t_{k}\right)\right)=\omega_{n}\left(\alpha_{n}\left(t_{1}\right)\left(A_{1}\right) \cdots \alpha_{n}\left(t_{k}\right)\left(A_{k}\right)\right)
$$

leads to a state $\tilde{\omega}_{n}$ on $\mathfrak{B}(\mathfrak{A})$.

We shall need the following properties of $\tilde{\omega}_{n}$ :

$$
\tilde{\omega}_{n}\left(g_{t}(P)\right)=\tilde{\omega}_{n}(P) \text { for all } P \in \mathfrak{B}(\mathfrak{A}) \text { and } t \in \boldsymbol{R} .
$$

(21) For every positive integer $k$ and every subset $A_{1}, \cdots, A_{k}$ of elements in $\mathfrak{A}$, the function $\left(t_{1}, \cdots, t_{k}\right) \rightarrow \tilde{\omega}_{n}\left(\left(A_{1}, t_{1}\right) \cdots\left(A_{k}, t_{k}\right)\right)$ is uniformly bounded on $\boldsymbol{R}^{k}$, in fact by $\Pi_{i}\left\|A_{i}\right\|$.

(22) Similarly, for every $k$ and $A_{1}, \cdots, A_{k}$ in $\mathfrak{A}_{0}$, the partial derivatives

$$
\partial \tilde{\omega}_{n}\left(\left(A_{1}, t_{1}\right) \cdots\left(A_{k}, t_{k}\right)\right)_{k} / \partial t_{i} \text { and } \partial^{2} \tilde{\omega}_{n}\left(\left(A_{1}, t_{1}\right) \cdots\left(A_{k}, t_{k}\right)\right) / \partial t_{i} \partial t_{j}
$$

are uniformly bounded on $\boldsymbol{R}^{k}$.

The contention (20) is clear, because, since $\alpha_{n}(t)$ defines a *automorphism group of $\mathfrak{A}$, the condition that $\omega_{n}$ is invariant under $\alpha_{n}(t)$ is equivalent to $\tilde{\omega}_{n}$ on $\mathfrak{B}(\mathfrak{R})$ being invariant under $g_{t}$. Indeed $\tilde{\omega}_{n}\left(g_{t}\left(\left(A_{1}, t_{1}\right) \ldots\right.\right.$ 
$\left.\left.\left(A_{k}, t_{k}\right)\right)\right)=\omega_{n}\left(\alpha_{n}(t)\left(\alpha_{n}\left(t_{1}\right)\left(A_{1}\right) \cdots \alpha_{n}\left(t_{k}\right)\left(A_{k}\right)\right)=\omega_{n}\left(\alpha_{n}\left(t_{1}\right)\left(A_{1}\right) \cdots \alpha_{n}\left(t_{k}\right)\left(A_{k}\right)\right)\right.$, so (20) follows by linearity.

The boundedness in (21) follows from (19). Indeed

$$
\begin{aligned}
& \left|\omega_{n}\left(\alpha_{n}\left(t_{1}\right)\left(A_{1}\right) \cdots \alpha_{n}\left(t_{k}\right)\left(A_{k}\right)\right)\right| \leqq\left\|\alpha_{n}\left(t_{1}\right)\left(A_{1}\right) \cdots \alpha_{n}\left(t_{k}\right)\left(A_{k}\right)\right\| \\
& \quad \leqq \prod_{i}\left\|\alpha_{n}\left(t_{i}\right)\left(A_{i}\right)\right\|=\prod_{i}\left\|A_{i}\right\|
\end{aligned}
$$

As for (22), we start with the case $k=1$. We have $\tilde{\omega}_{n}\left(\left(A_{1}, t_{1}\right)\right)=$ $\omega_{n}\left(\alpha_{n}\left(t_{1}\right)\left(A_{1}\right)\right)=\omega_{n}\left(A_{1}\right)$ so the derivatives vanish identically. For $k \geqq 2$ and $A_{1}, \cdots, A_{k} \in \mathfrak{A}_{0}$

$$
\begin{aligned}
& \left|\partial \tilde{\omega}_{n}\left(\left(A_{1}, t_{1}\right) \cdots\left(A_{k}, t_{k}\right)\right) / \partial t_{i}\right|=\left|\omega_{n}\left(\cdots \alpha_{n}\left(t_{i}\right)\left(\delta_{n}\left(A_{i}\right)\right) \cdots\right)\right| \\
& \quad \leqq \prod_{p \neq i}\left\|A_{p}\right\|\left\|\delta_{n}\left(A_{i}\right)\right\| .
\end{aligned}
$$

The right hand side is bounded since $\delta_{n}\left(A_{i}\right) \rightarrow \delta\left(A_{i}\right)$. (It is important at this point that the definition of inner limit *-derivations involves sequences rather than nets, since convergent nets need not be bounded. We deviate from the definition in [5] at this point.)

Similarly we have for $i \neq j$

$$
\left|\partial^{2} \omega_{n}\left(\left(A_{1}, t_{1}\right) \cdots\left(A_{k}, t_{k}\right)\right) / \partial t_{i} \partial t_{j}\right| \leqq \prod_{p \neq i, j}\left\|A_{p}\right\|\left\|\delta_{n}\left(A_{i}\right)\right\|\left\|\delta_{n}\left(A_{j}\right)\right\|
$$

Considering finally $\partial^{2} / \partial t_{i}^{2}$ we will assume for the sake of simplicity that $i=1$ and $k=2$.

$$
\begin{aligned}
\partial \tilde{\omega}_{n}\left(\left(A_{1}, t_{1}\right)\left(A_{2}, t_{2}\right)\right) / \partial t_{1} & \left.=\omega_{n}\left(\alpha_{n}\left(t_{1}\right) \delta_{n}\left(A_{1}\right)\right) \alpha_{n}\left(t_{2}\right)\left(A_{2}\right)\right) \\
& =\omega_{n}\left(\delta_{n}\left(A_{1}\right) \alpha_{n}\left(t_{2}-t_{1}\right)\left(A_{2}\right)\right),
\end{aligned}
$$

and

$$
\partial^{2} \tilde{\omega}_{n}\left(\left(A_{1}, t_{1}\right)\left(A_{2}, t_{2}\right)\right) / \partial t_{1}^{2}=-\omega_{n}\left(\delta_{n}\left(A_{1}\right) \alpha_{n}\left(t_{2}-t_{1}\right)\left(\delta_{n}\left(A_{2}\right)\right)\right),
$$

and hence

$$
\left|\partial^{2} \tilde{\omega}_{n}(\quad) / \partial t_{1}^{2}\right| \leqq\left\|\delta_{n}\left(A_{1}\right)\right\|\left\|\delta_{n}\left(A_{2}\right)\right\| \text {. }
$$

Since the right hand side is bounded, the proof is completed.

We have checked that for fixed $A_{1}, \cdots, A_{k}$ in $\mathfrak{A}_{0}$, the set of functions $K=\left\{\left(t_{1}, \cdots, t_{k}\right) \rightarrow \tilde{\omega}_{n}\left(\left(A_{1}, t_{1}\right) \cdots\left(A_{k}, t_{k}\right)\right): n=1,2, \cdots\right\}$ is bounded in (the Frechet topology of) $C^{2}\left(\boldsymbol{R}^{k}\right)$. By a classical result [23, Theorem 14.4], based on the Arzela-Ascoli theorem, we conclude that $K$ is relatively compact in $C^{1}\left(\boldsymbol{R}^{k}\right)$. Hence, there is a subsequence (depending on $\left.\left(A_{1}, \cdots, A_{k}\right)\right)$ of $K$ which converges in the $C^{1}\left(\boldsymbol{R}^{k}\right)$ topology. Defining $f_{n}\left(t_{1}, \cdots, t_{k}\right)=\tilde{\omega}_{n}\left(\left(A_{1}, t_{1}\right) \cdots\left(A_{k}, t_{k}\right)\right)$ we have, for the subsequence $\left\{n_{p}\right\}$ in question, that each of the sequences $\left\{f_{n_{p}}\right\},\left\{\partial f_{n_{p}} / \partial t_{i}\right\}$ for $1 \leqq i \leqq k$ are convergent the convergence being uniform on every compact subset of 
$\boldsymbol{R}^{k}$, and so in particular pointwise. (We note that the Arzela-Ascoli theorem has been used in a similar context by Ruskai [19].) There is a function $G\left(A_{1}, \cdots, A_{k} ; t_{1}, \cdots, t_{k}\right)$ belonging to $C^{1}\left(\boldsymbol{R}^{k}\right)$ in the $t$-variable such that

$$
\lim _{n_{p}} \tilde{\omega}_{n_{p}}\left(\left(A_{1}, t_{1}\right) \cdots\left(A_{k}, t_{k}\right)\right)=G\left(A_{1}, \cdots, A_{k_{j}}, t_{1}, \cdots, t_{k}\right)
$$

and

$$
\lim _{n_{p}} \partial \tilde{\omega}_{n_{p}}\left(\left(A_{1}, t_{1}\right) \cdots\left(A_{k}, t_{k}\right)\right) / \partial t_{i}=\partial G\left(A_{2}, \cdots, A_{k} ; t_{1}, \cdots, t_{k}\right) / \partial t_{i},
$$

again uniformly for $t$ in compacts.

We now define a linear functional $\tilde{\boldsymbol{\omega}}$ on $\mathfrak{B}\left(\mathfrak{A}_{0}\right)$ by

$$
\tilde{\omega}\left(\left(A_{1}, t_{1}\right) \cdots\left(A_{k}, t_{k}\right)\right)=G\left(A_{1}, \cdots, A_{k} ; t_{1}, \cdots, t_{k}\right) \text {. }
$$

We claim that $\tilde{\omega}$ extends to a state on $\mathfrak{B}(\mathfrak{U})$ which satisfies properties (i) and (ii) of Theorem 1 together with property (8) of Remark 3(b).

We first extend $\tilde{\omega}$ to a linear functional on $\mathfrak{B}(\mathfrak{U})$. By linearity it is then enough to define the extension (also denoted by $\tilde{\omega}$ ) on arbitrary elements $Q=\left(A_{1}, t_{1}\right) \cdots\left(A_{k}, t_{k}\right)$ for $A_{1}, \cdots, A_{k}$ in $\mathfrak{A}$. Given such an element, we pick sequences $\left\{A_{1, q}\right\} \cdots\left\{A_{k, q}\right\}$ of elements in $\mathfrak{A}_{0}$ such that $\lim _{q} A_{i, q}=A_{i}$ for $i=1, \cdots, k$. We then verify that the corresponding sequence of scalars $\left\{\tilde{\omega}\left(\left(A_{1, q}, t_{1}\right) \cdots\left(A_{k, q}, t_{k}\right)\right)\right\}_{q}$ is Cauchy. For this purpose we combine (23) and (24) and get

$$
\begin{aligned}
& \left|\tilde{\omega}\left(\left(A_{1, q}, t_{1}\right) \cdots\left(A_{k, q}, t_{k}\right)\right)-\tilde{\omega}\left(\left(A_{1, r}, t_{1}\right) \cdots\left(A_{k, r}, t_{k}\right)\right)\right| \\
& \quad \leqq \sum_{i=1}^{k} \prod_{j=1}^{i-1}\left\|A_{j, r}\right\|\left\|A_{i, q}-A_{i, r}\right\| \prod_{j=i+1}^{k}\left\|A_{j, q}\right\| .
\end{aligned}
$$

The right hand side tends to zero as $q, r \rightarrow \infty$ by assumption.

By $(24)$ we have $\tilde{\omega}\left(Q^{*} Q\right)=\lim _{n_{p}} \tilde{\omega}_{n_{p}}\left(Q^{*} Q\right) \geqq 0$ for all $Q \in \mathfrak{B}(\mathfrak{R})$, where $\left\{n_{p}\right\}$ is a subsequence of the integers which depend on $Q^{*} Q$. The estimates (27) also imply, together with the usual $3 \varepsilon$ argument, that for every $A_{1}, \cdots, A_{k}$ in $\mathfrak{A}$ (not only $\mathfrak{U}_{0}$ ) there is a subsequence $\left\{n_{p}\right\}$ such that the convergence (24) holds.

Combination of this with definition (26) now yields the conclusion (13) of Lemma 2.

Condition (i) of Theorem 1 follows immediately from this:

$$
\begin{aligned}
\tilde{\omega}\left(g_{t}(Q)\right) & =\lim _{n_{p}} \tilde{\omega}_{n_{p}}\left(\left(A_{1}, t_{1}+t\right) \cdots\left(A_{k}, t_{k}+t\right)\right) \\
& =\lim _{n_{p}} \omega_{n_{p}}\left(\alpha_{n_{p}}(t)\left\{\alpha_{n_{p}}\left(t_{1}\right)\left(A_{1}\right) \cdots \alpha_{n_{p}}\left(t_{k}\right)\left(A_{k}\right)\right\}\right) \\
& =\lim _{n_{p}} \omega_{n_{p}}\left(\alpha_{n_{p}}\left(t_{1}\right)\left(A_{1}\right) \cdots \alpha_{n_{p}}\left(t_{k}\right)\left(A_{k}\right)\right) \\
& =\lim _{n_{p}} \tilde{\omega}_{n_{p}}\left(\left(A_{1}, t_{1}\right) \cdots\left(A_{k}, t_{k}\right)\right)=\tilde{\omega}(Q)
\end{aligned}
$$

for all $t \in \boldsymbol{R}$. This completes the proof of (i). 
Property (ii) follows from the observation that $G\left(A_{1}, \cdots, A_{k}, \cdots\right)$ in (24) is continuous, as a uniform limit on compacts of continuous functions.

Finally the estimate (8) of Remark 3(b) is satisfied for each of the states $\tilde{\omega}_{n_{p}}$, and hence for the limit state $\tilde{\omega}$. We claim that $\tilde{\omega}$ is an extension of the state $\omega$, which was defined as the weak-* limit on $\mathfrak{A}$ of the sequence $\left\{\omega_{n}\right\}$, cf. (18). To see this we note that for $A \in \mathfrak{A}$,

$$
\tilde{\omega}((A, 0))=\lim _{n} \tilde{\omega}_{n}((A, 0))=\lim _{n} \omega_{n}(A)=\omega(A) .
$$

This completes the proof of Lemma 2.

Proof of Lemma 3. We now apply the generalized GNS construction which is established in Theorem 1 , to the state $\tilde{\omega}$. We get a Hilbert space $\mathscr{K}$, a bounded (by Remark $3(\mathrm{~b})$ ) representation $\pi$ of $\mathfrak{B}(\mathfrak{A})$ on $\mathscr{K}$, a continuous unitary representation $U(t)$, and a vector $\xi \in \mathscr{K}$ such that $\tilde{\boldsymbol{\omega}}(Q)=(\xi, \pi(Q) \xi)$ for all $Q \in \mathfrak{B}(\mathfrak{R})$, and properties (c) and (d) are satisfied. We let $\pi_{0}$ denote the restriction to $\mathfrak{A}$ of $\pi$. The state $\omega_{0}(A)=\left(\xi, \pi_{0}(A) \xi\right)$ then coincides with the limit state $\omega$. For by $(13) \omega_{0}(A)=\tilde{\omega}((A, 0))=$ $\lim _{n} \tilde{\omega}_{n}((A, 0))=\omega(A)$.

Moreover, the extension $\omega^{\prime}$ say of $\omega_{0}=\omega$ given by (3) in Theorem 2 coincides with $\tilde{\boldsymbol{\omega}}$. Indeed,

$$
\begin{aligned}
\omega^{\prime}\left(\left(A_{1}, t_{1}\right) \cdots\left(A_{k}, t_{k}\right)\right) & =\left(\xi, \pi_{0}\left(A_{1}\right) U\left(t_{2}-t_{1}\right) \cdots U\left(t_{k}-t_{k-1}\right) \pi_{0}\left(A_{k}\right) \xi\right) \\
& =\left(\xi, U\left(t_{1}\right) \pi_{0}\left(A_{1}\right) U\left(-t_{1}\right) \cdots U\left(t_{k}\right) \pi_{0}\left(A_{k}\right) U\left(-t_{k}\right) \xi\right) \\
& =\left(\xi, \pi\left(g_{t_{1}} A_{1}\right) \cdots \pi\left(g_{t_{k}} A_{k}\right) \xi\right) \\
& =\left(\xi, \pi\left(\left(A_{1}, t_{1}\right) \cdots\left(A_{k}, t_{k}\right)\right) \xi\right)=\tilde{\omega}\left(\left(A_{1}, t_{1}\right) \cdots\left(A_{k}, t_{k}\right)\right) .
\end{aligned}
$$

Given elements $A, B \in \mathfrak{A}_{0}$ it follows from (25) that the function $t \rightarrow \widetilde{\boldsymbol{\omega}}(A(B, t))$ is of class $C^{1}(\boldsymbol{R})$. This formula also gives a recipe for $(d / d t) \tilde{\omega}(A(B, t))$. Corresponding to $A$ and $B$ there is a subsequence $\left\{n_{p}\right\}$ such that

$$
\begin{aligned}
\left.\frac{d}{d t} \tilde{\omega}(A(B, t))\right|_{t=0} & =\left.\lim _{n_{p}} \frac{d}{d t} \tilde{\omega}_{n_{p}}(A(B, t))\right|_{t=0}=\left.\lim _{n_{p}} \frac{d}{d t} \omega_{n_{p}}\left(A \alpha_{n_{p}}(t)(B)\right)\right|_{t=0} \\
& =\lim _{n_{p}} \omega_{n_{p}}\left(A \delta_{n_{p}}(B)\right)=\omega_{0}(A \delta(B)),
\end{aligned}
$$

since $\left\|\delta_{n_{p}}(B)-\delta(B)\right\| \rightarrow 0$ and $\omega_{n_{p}} \rightarrow \omega_{0}$ in the weak-* topology. Specifically,

$$
\begin{aligned}
& \left|\omega_{n_{p}}\left(A \delta_{n_{p}}(B)\right)-\omega_{0}(A \delta(B))\right| \\
& \quad \leqq\left|\omega_{n_{p}}\left(A \delta_{n_{p}}(B)\right)-\omega_{n_{p}}(A \delta(B))\right|+\left|\omega_{n_{p}}(A \delta(B))-\omega_{0}(A \delta(B))\right| \\
& \quad \leqq|| A|||| \delta_{n_{p}}(B)-\delta(B) \|+\left|\left(\omega_{n_{p}}-\omega_{0}\right)(A \delta(B))\right| \rightarrow 0 .
\end{aligned}
$$

Consequently, 


$$
\tilde{\omega}(A(B, t))=\omega_{0}(A B)+t \omega_{0}(A \delta(B))+o(t) .
$$

As a corollary to (29) we get, using $\omega_{n} \alpha_{n}(t)=\omega_{n}$ and $\omega_{0}=\omega$,

$$
\omega(A \delta(B))=\lim _{n_{p}} \omega_{n_{p}}\left(A \delta_{n_{p}}(B)\right)=-\lim _{n_{p}} \omega_{n_{p}}\left(\delta_{n_{p}}(A) B\right)=-\omega(\delta(A) B) .
$$

Now suppose that $\delta$ is also regular. Then for every $k$ and every $A_{1}, \cdots, A_{k} \in \mathfrak{Q}_{0}$ we have

$$
\sup \left\{\left|D_{t}^{3} \tilde{\boldsymbol{\omega}}_{n}\left(\left(A_{1}, t_{1}\right) \cdots\left(A_{k}, t_{k}\right)\right)\right|: n=1,2, \cdots,\left(t_{1}, \cdots, t_{k}\right) \in \boldsymbol{R}^{k}\right\}<\infty .
$$

The symbol $D_{t}^{3}$ stands for a differential monomial of degree 3 in the variables $\left(t_{1}, \cdots, t_{k}\right)$, i.e., $\partial^{3} / \partial t_{i_{1}} \partial t_{i_{2}} \partial t_{i_{3}}, 1 \leqq i_{1}, i_{2}, i_{3} \leqq k$.

To show boundedness of $D_{t}^{3} \tilde{\omega}_{n}$, it is necessary to treat the different cases separately.

Case 1: $k \geqq 3$ and $1 \leqq i_{1} \leqq i_{2} \leqq i_{3} \leqq k$. Then

$$
D_{t}^{3} \tilde{\omega}_{n}(\quad)=\omega_{n}\left(B_{1}, \cdots, B_{k}\right) \quad \text { where } \quad B_{i_{p}}=\alpha_{n}\left(t_{i_{p}}\right)\left(\delta_{n}\left(A_{i_{p}}\right)\right)
$$

for $p=1,2,3$ and $B_{j}=\alpha_{n}\left(t_{j}\right)\left(A_{j}\right)$ for $j$ different from $i_{1}, i_{2}, i_{3}$. Clearly then

$$
\left|D_{t}^{3} \tilde{\omega}_{n}(\quad)\right| \leqq\left(\prod_{i \neq i_{1}, i_{2}, i_{3}}\left\|A_{j}\right\|\right)\left\|\delta_{n}\left(A_{i_{1}}\right)||\right\| \delta_{n}\left(A_{i_{2}}\right)\|\| \delta_{n}\left(A_{i_{3}}\right) \| \text {. }
$$

Case 2: $k \geqq 2$ and $1 \leqq i_{1}=i_{2}=i \leqq i_{3} \leqq k$. Then $D_{t}^{3} \tilde{\boldsymbol{\omega}}_{n}(\quad)=$ $\omega_{n}\left(B_{1}, \cdots, B_{k}\right) \quad$ where $B_{i}=\alpha_{n}\left(t_{i}\right)\left(\delta_{n}^{2}\left(A_{i}\right)\right), \quad B_{i_{3}}=\alpha_{n}\left(t_{i_{3}}\right)\left(\delta_{n}\left(A_{i_{3}}\right)\right)$ and $B_{j}=$ $\alpha_{n}\left(t_{j}\right)\left(A_{j}\right)$ for $j$ different from $i$ and $i_{3}$. Then

$$
\left|D_{t}^{3} \tilde{\omega}_{n}(\quad)\right| \leqq\left(\prod_{j \neq i, i_{3}} \| A_{j}||\right)\left\|\delta_{n}^{2}\left(A_{i}\right)\right\|\left\|\delta_{n}\left(A_{i_{3}}\right)\right\|,
$$

and the right hand side is bounded since $\delta$ is assumed regular.

Case 3: $i_{1}=i_{2}=i_{3}=i$ and $1 \leqq i \leqq k$. Then $D_{t}^{3} \tilde{\omega}_{n}(\quad)=\omega_{n}\left(B_{1}, \cdots, B_{k}\right)$ where $B_{j}=\alpha_{n}\left(t_{j}\right)\left(A_{j}\right)$ for $j \neq i$ and $B_{i}=\alpha_{n}\left(t_{i}\right)\left(\delta_{n}^{3}\left(A_{i}\right)\right)=\delta_{n}\left(\alpha_{n}\left(t_{i}\right)\left(\delta_{n}^{2}\left(A_{i}\right)\right)\right)$. We now put $C_{i}=\alpha_{n}\left(t_{i}\right)\left(\delta_{n}^{2}\left(A_{i}\right)\right)$ and have

$$
\begin{aligned}
D_{t}^{3} \tilde{\omega}_{n}(\quad) & =\omega_{n}\left(B_{1} \cdots B_{i-1} \delta_{n}\left(C_{i}\right) B_{i+1} \cdots B_{k}\right) \\
& =\left.\frac{d}{d t} \omega_{n}\left(B_{1} \cdots B_{i-1} \alpha_{n}(t)\left(C_{i}\right) B_{i+1} \cdots B_{k}\right)\right|_{t=0} \\
& =\left.\frac{d}{d t} \omega_{n}\left(\left[\alpha_{n}(-t)\left(B_{1} \cdots B_{i-1}\right)\right] C_{i}\left[\alpha_{n}(-t)\left(B_{i+1} \cdots B_{k}\right)\right]\right)\right|_{t=0} \\
& =-\omega_{n}\left(\delta_{n}\left(B_{1} \cdots B_{i-1}\right) C_{i}\left(B_{i+1} \cdots B_{k}\right)\right) \\
& =-\omega_{n}\left(B_{1} \cdots B_{i-1} C_{i} \delta_{n}\left(B_{i+1} \cdots B_{k}\right)\right) .
\end{aligned}
$$

Using finally $\delta_{n}\left(B_{1} \cdots B_{i-1}\right)=\sum_{j=1}^{i-1} B_{1} \cdots B_{j-1} \delta_{n}\left(B_{j}\right) B_{j+1} \cdots B_{i-1}$ and the similar identity for $\delta_{n}\left(B_{i+1} \cdots B_{k}\right)$ we get 


$$
\left|D_{t}^{3} \tilde{\omega}_{n}(\quad)\right| \leqq \sum_{j \neq i}\left\|\delta_{n}\left(A_{j}\right)\right\|\left(\prod_{p \neq j, i}\left\|A_{p}\right\|\right)\left\|\delta_{n}^{2}\left(A_{i}\right)\right\| \text {. }
$$

Again the right hand side is bounded when $\delta$ is regular.

A new application of [23, Theorem 14.4], now using that boundedness in $C^{3}\left(\boldsymbol{R}^{k}\right)$ implies relative compactness in $C^{2}\left(\boldsymbol{R}^{k}\right)$, shows that for each $A_{1}, \cdots, A_{k} \in \mathfrak{A}_{0}$ we may choose a subsequence $\left\{n_{p}\right\}$ of the integers such that (24) and (25) hold in addition to

$$
\lim _{n_{p}} D_{t}^{2} \widetilde{\omega}_{n_{p}}\left(\left(A_{1}, t_{1}\right) \cdots\left(A_{k}, t_{k}\right)\right)=D_{t}^{2} G\left(A_{1}, \cdots, A_{k} ; t_{1}, \cdots, t_{k}\right)
$$

for every second degree differential monomial $D_{t}^{2}$. Consequently, the Green's function $G\left(A_{1}, \cdots, A_{k_{j}} \cdots\right)$ belongs to $C^{2}\left(\boldsymbol{R}^{k}\right)$.

A computation similar to the one leading from (25) to (30) gives

$$
\tilde{\omega}(A(B, t))=\omega(A B)+t \omega(A \delta(B))+t^{2} / 2 \omega\left(A \delta^{2}(B)\right)+o\left(t^{2}\right)
$$

for the case where $\delta$ is regular. (Recall that the Green's function $G(A, B, \cdots)$ is of class $C^{2}$ when $\delta$ is regular.)

Therefore

$$
\tilde{\omega}\left(\left(A^{*}, t\right) A\right)+\tilde{\omega}\left(A^{*}(A, t)\right)=2 \omega\left(A^{*} A\right)-t^{2} \omega\left(\delta\left(A^{*}\right) \delta(A)\right)+o\left(t^{2}\right)
$$

and

$$
\tilde{\omega}\left(\left(A^{*}, t\right) \delta(A)\right)+\omega\left(\delta\left(A^{*}\right)(A, t)\right)=2 t \omega\left(\delta\left(A^{*}\right) \delta(A)\right)+o(t) .
$$

We now substitute this into the following expression and get for $A \in \mathfrak{Q}_{0}$ :

$$
\begin{aligned}
&\left\|U(t) \pi_{0}(A) \xi-\pi_{0}(A) \xi-t \pi_{0}(\delta(A)) \xi\right\|_{\mathscr{S}}^{2} \\
&=\tilde{\omega}\left(((A, t)-A-t \delta(A))^{*}((A, t)-A-t \delta(A))\right) \\
&=\tilde{\omega}\left(\left(\left(A^{*}, t\right)-A^{*}-t \delta\left(A^{*}\right)\right)((A, t)-A-t \delta(A))\right) \\
&=\tilde{\omega}\left(\left(A^{*}, t\right)(A, t)\right)+\omega\left(A^{*} A\right)+t^{2} \omega\left(\delta\left(A^{*}\right) \delta(A)\right) \\
&-\left[\tilde{\omega}\left(\left(A^{*}, t\right) A\right)+\tilde{\omega}\left(A^{*}(A, t)\right)\right] \\
&-t\left[\tilde{\omega}\left(\left(A^{*}, t\right) \delta(A)\right)+\tilde{\omega}\left(\delta\left(A^{*}\right)(A, t)\right)\right] \\
&+t\left[\omega\left(A^{*} \delta(A)\right)+\omega\left(\delta\left(A^{*}\right) A\right)\right] \\
&= \tilde{\omega}\left(\left(A^{*} A, t\right)\right)+\omega\left(A^{*} A\right)+t^{2} \omega\left(\delta\left(A^{*}\right) \delta(A)\right) \\
&-2 \omega\left(A^{*} A\right)+t^{2} \omega\left(\delta\left(A^{*}\right) \delta(A)\right)+o\left(t^{2}\right) \\
&-t\left[2 t \omega\left(\delta\left(A^{*}\right) \delta(A)\right)+o(t)\right]=o\left(t^{2}\right),
\end{aligned}
$$

since $\tilde{\omega}\left(\left(A^{*} A, t\right)\right)=\omega\left(A^{*} A\right)$ and $t o(t)=o\left(t^{2}\right)$. Consequently,

$$
\begin{aligned}
\lim _{t \rightarrow 0 \pm}\left\|t^{-1}\left(U(t) \pi_{0}(A) \xi-\pi_{0}(A) \xi\right)-\pi_{0}(\delta(A)) \xi\right\|_{\mathscr{H}}^{2} \\
\quad=\lim _{t \rightarrow 0 \pm} t^{-2}\left\|U(t) \pi_{0}(A) \xi-\pi_{0}(A) \xi-t \pi_{0}(\delta(A)) \xi\right\|_{\mathscr{\varkappa}}^{2} \\
\quad=\lim _{t \rightarrow 0 \pm} t^{-2} o\left(t^{2}\right)=0 .
\end{aligned}
$$


Consequently, the vector $\pi_{0}(A) \xi$ belongs to the domain of $H$ and

$$
i H\left(\pi_{0}(A) \xi\right)=\pi_{0}(\delta(A)) \xi \text {. }
$$

If $A$ is now an arbitrary element in the domain of $\delta$, then there is a sequence $\left\{A_{n}\right\}$ of elements in $\mathfrak{A}_{0}$ such that $A_{n} \rightarrow A$ and $\delta\left(A_{n}\right) \rightarrow \delta(A)$. Since (32) holds for each $A_{n}$ and $\pi_{0}$ is continuous, we get $\pi_{0}\left(A_{n}\right) \xi \rightarrow \pi_{0}(A) \xi$ and $i H\left(\pi_{0}\left(A_{n}\right) \xi\right) \rightarrow \pi_{0}(\delta(A)) \xi$. Since $H$ is self-adjoint, and in particular closed, we conclude that $\pi_{0}(A) \xi$ is in the domain of $H$ and that (32) holds for $A$.

q.e.d.

Proof of Theorem 4. Let $\delta$ be an inner limit derivation in $\mathfrak{A}$, and let $\left\{H_{n}\right\}$ be an approximating sequence of hermitian elements in $\mathfrak{A}$. Let $\tau$ denote a trace state on $\mathfrak{A}$ (i.e., $\tau(A B)=\tau(B A), \tau\left(A^{*} A\right) \geqq 0, \tau(I)=1$ ) and define a sequence $\left\{\omega_{n}\right\}$ of states on $\mathfrak{A}$ by

$$
\omega_{n}(A)=\tau\left(e^{-\beta H_{n}} A\right) / \tau\left(e^{-\beta H_{n}}\right) \text { for } \quad A \in \mathfrak{A} .
$$

It is immediate that $\omega_{n}$ is a $\beta$-KMS state with respect to $\alpha_{n}(t)=e^{i t H_{n}}$. $e^{-i t H_{n}}$ for each $n$; so that (12) in particular is satisfied.

We let $\tilde{\boldsymbol{\omega}}$ denote the state on $\mathfrak{B}(\mathfrak{H})$ which is given by Lemma 2. The dynamical system induced by this state is denoted by $\left(\pi_{0}, \mathfrak{A}, \mathscr{K}, \xi, U(t)\right)$. The Hamiltonian $H$ given by $U(t)=e^{i t H}$ satisfies (14) of Lemma 3.

It remains to show that $\tilde{\omega}$ is a $\beta$-KMS state on $\mathfrak{B}(\mathfrak{U})$. This is quite simple in the present context. We will verify condition $\left(g^{\prime}\right)$ of Definition 3 for every pair $P, Q$ of elements in $\mathfrak{B}(\mathfrak{A})$ and every entire function $\varphi$ on $\boldsymbol{R}$ such that $\hat{\varphi} \in \mathscr{D}$. We first claim that the states $\omega_{n}$ are constructed in such a way that the identity

$$
\int \varphi(t) \tilde{\omega}_{n}\left(P g_{t}(Q)\right) d t=\int \varphi(t+i \beta) \tilde{\omega}_{n}\left(g_{t}(Q) P\right) d t
$$

holds for all $P, Q$ and $\varphi$ as above.

Again, by linearity, it is enough to verify the identities for elements $P=\left(A_{1}, s_{1}\right) \cdots\left(A_{k}, s_{k}\right) \quad Q=\left(B_{1}, t_{1}\right) \cdots\left(B_{f}, t_{f}\right)$. Using that $\alpha_{n}(\cdot)$ is a *-automorphism of $\mathfrak{A}$, we note that the elements

$$
A=\alpha_{n}\left(s_{1}\right)\left(A_{1}\right) \cdots \alpha_{n}\left(s_{k}\right)\left(A_{k}\right) \text { and } B=\alpha_{n}\left(t_{1}\right)\left(B_{1}\right) \cdots \alpha_{n}\left(t_{f}\right)\left(B_{f}\right)
$$

both belong to $\mathfrak{A}$, so that Lemma 1 applies, and

$$
\begin{aligned}
& \int \varphi(t) \tilde{\omega}_{n}\left(P g_{t}(Q)\right) d t=\int \varphi(t) \omega_{n}\left(A \alpha_{n}(t)(B)\right) d t \\
& \quad=\int \varphi(t+i \beta) \omega_{n}\left(\alpha_{n}(t)(B) A\right) d t=\int \varphi(t+i \beta) \tilde{\omega}_{n}\left(\left(g_{t}(Q)\right) P\right) d t .
\end{aligned}
$$

This concludes the proof of (33). 
Now let $\varphi, P$ and $Q$ be arbitrary, and assume first that $P, Q \in \mathfrak{B}\left(\mathfrak{A}_{0}\right)$. Then by (24) and (26) there is a subsequence $\left\{n_{p}\right\}$ such that $\tilde{\omega}_{n_{p}}\left(P g_{t}(Q)\right) \rightarrow$ $\tilde{\omega}\left(P g_{t}(Q)\right)$ and $\tilde{\omega}_{n_{p}}\left(g_{t}(Q) P\right) \rightarrow \tilde{\omega}\left(g_{t}(Q) P\right)$ uniformly to $t$ in compact subsets of $\boldsymbol{R}$. We conclude that (33) holds, now with $\tilde{\omega}_{n}$ replaced by $\tilde{\omega}$.

Finally, we let $P$ and $Q$ be arbitrary elements in $\mathfrak{B}(\mathfrak{A})$. By (27) we conclude that there are sequences $\left\{P_{q}\right\}$ and $\left\{Q_{q}\right\}$ in $\mathfrak{B}\left(\mathfrak{A}_{0}\right)$ such that $\tilde{\omega}\left(P_{q} g_{t}\left(Q_{q}\right)\right) \rightarrow \tilde{\omega}\left(P g_{t}(Q)\right)$ and $\tilde{\omega}\left(g_{t}\left(Q_{q}\right) P_{q}\right) \rightarrow \tilde{\omega}\left(g_{t}(Q) P\right)$ as $q \rightarrow \infty$, in fact uniformly in $t$. The desired identity

$$
\int \varphi(t) \tilde{\boldsymbol{\omega}}\left(P g_{t}(Q)\right) d t=\int \varphi(t+i \beta) \tilde{\boldsymbol{\omega}}\left(g_{t}(Q) P\right) d t
$$

q.e.d.

CoRollary 3. Application of Theorem 3 to the states $\tilde{\boldsymbol{\omega}}_{\beta}$ constructed in Theorem 4 shows that the multiple time Green's function $G\left(A_{1}, \cdots, A_{k}\right.$; $\left.t_{1}, \cdots, t_{k}\right)=\tilde{\omega}_{\beta}\left(\left(A_{1}, t_{1}\right) \cdots\left(A_{k}, t_{k}\right)\right)$ has an analytic continuation $G\left(A_{1}, \cdots, A_{k}\right.$; $z_{1}, \cdots, z_{k}$ ) to the tube domain $0<\operatorname{Im} z_{1}<\cdots<\operatorname{Im} z_{k}<\beta$. (Such analytic continuation has been considered in special cases by various authors [1], [8], [19] and [24].)

REMARK 5. It follows from [3, Theorem 3.1] that for given $A_{1}, \cdots, A_{k}$ in $\mathfrak{A}$ the analytic function $G\left(A_{1}, \cdots, A_{k} ; z_{1}, \cdots, z_{k}\right)$ has a uniformly bounded and continuous extension to the closed tube-domain $0 \leqq \operatorname{Im} z_{1} \leqq \cdots \leqq$ $\operatorname{Im} z_{k} \leqq \beta / 2$. Moreover the estimate

$$
\left|G\left(A_{1}, \cdots, A_{k} ; z_{1}, \cdots, z_{k}\right)\right| \leqq\left\|A_{1}\right\| \cdots\left\|A_{k}\right\|\|\xi\|^{2}
$$

holds in this domain.

Proof of Theorem 5. Let $\delta$ be an inner limit derivative in $\mathfrak{A}$, and let $\left\{H_{n}\right\}$ be an approximating sequence of hermitian elements in $\mathfrak{A}$. The corresponding sequence of automorphism groups is denoted by $\left\{\alpha_{n}(t)\right\}$ as above. It is pointed out in [15] that $\alpha_{n}(t)$ has a ground state $\omega_{n}$ for each $n$. (We may assume that the spectrums $\sigma\left(H_{n}\right) \subset[0, \infty]$ and $0 \in \sigma\left(H_{n}\right)$ for all $n$. If this is not satisfied we may replace $H_{n}$ by $H_{n}-\lambda_{n} I$ where $\lambda_{n}=\inf \sigma\left(H_{n}\right)$. The $C^{*}$-algebra $C^{*}\left(H_{n}\right)$ generated by $H_{n}$ and $I$ is isomorphic to $C\left(\sigma\left(H_{n}\right)\right)$. On $C^{*}\left(H_{n}\right)$ we let $\omega_{n}$ be the state corresponding to point evaluation at zero, $f \rightarrow f(0)$, and then extend $\omega_{n}$ to a state on $\mathfrak{A}_{\text {.) }}$

It follows from [15] that $\omega_{n} \alpha_{n}(t)=\omega_{n}$ for all $n$. This can also be seen directly as follows: For every $A \in \mathfrak{A},\left|\omega_{n}\left(A H_{n}\right)\right|^{2} \leqq \omega_{n}\left(A A^{*}\right) \omega\left(H_{n}^{2}\right)=0$ since $\omega\left(H_{n}^{2}\right)=\hat{H}_{n}(0)^{2}=0$. Quite similarly, $\omega_{n}\left(H_{n} A\right)=0$ for all $A$. Consequently, $\omega_{n}\left(\left(\operatorname{ad} H_{n}\right)^{k}(A)\right)=0$ for all $k=1,2, \cdots$ Hence

$$
\omega_{n}\left(\alpha_{n}(t)(A)\right)=\sum_{k=0}^{\infty}(i t)^{k} / k ! \quad \omega_{n}\left(\left(\operatorname{ad} H_{n}\right)^{k}(A)\right)=\omega_{n}(A) .
$$


Whence Lemma 2 ensures the existence of the limit state $\tilde{\omega}$ on $\mathfrak{B}(\mathfrak{A})$ satisfying (13). By Lemma 3 , the corresponding Hamiltonian $H$ satisfies (14). It remains to show that $\tilde{\omega}$ is a ground state on $\mathfrak{B}(\mathfrak{A})$; i.e., that for every $k$ and every set $A_{1}, \cdots, A_{k}$ of elements in $\mathfrak{A}$ there exists an analytic function $F\left(z_{1}, \cdots, z_{k}\right)$ in the region $\operatorname{Im} z_{1}<\cdots<\operatorname{Im} z_{k}$ with boundary values

$$
F\left(t_{1}, \cdots, t_{k}\right)=\tilde{\omega}\left(\left(A_{1}, t_{1}\right) \cdots\left(A_{k}, t_{k}\right)\right)
$$

for all $t \in \boldsymbol{R}^{k}$.

But the existence of $F$ is quite clear from (3) applied to $\tilde{\boldsymbol{\omega}}$ once we have shown that the spectrum of $H$ is non-negative, i.e., $H \geqq 0$. We show first that

$$
(\pi(Q) \xi, H \pi(Q) \xi) \geqq 0 \text { for all } Q \in \mathfrak{B}\left(\mathfrak{A}_{0}\right) .
$$

Using (25) we observe that for every $Q \in \mathfrak{B}\left(\mathfrak{H}_{0}\right)$ there exists a sequence $\left\{n_{p}\right\}$ of integers such that

$$
(\pi(Q) \xi, i H \pi(Q) \xi)=\left.(d / d t) \tilde{\boldsymbol{\omega}}\left(Q^{*} g_{t}(Q)\right)\right|_{t=0}=\left.\lim _{n_{p}}(d / d t) \tilde{\omega}_{n_{p}}\left(Q^{*} g_{t}(Q)\right)\right|_{t=0} .
$$

Therefore it is enough to show that $-\left.i(d / d t) \tilde{\omega}_{n}\left(Q^{*} g_{t}(Q)\right)\right|_{t=0} \geqq 0$ for all $n$.

Let $Q \in \mathfrak{B}\left(\mathfrak{H}_{0}\right)$ be given. Suppose $Q=\sum_{q} Q_{q}$ (finite sum) with $Q_{q}=$ $\left(A_{1, q}, t_{1, q}\right) \cdots\left(A_{k, q}, t_{k, q}\right)$. Now put

$$
A_{q(n)}=\alpha_{n}\left(t_{1, q}\right)\left(A_{1, q}\right) \cdots \alpha_{n}\left(t_{k, q}\right)\left(A_{k, q}\right) \quad \text { and } \quad A_{(n)}=\sum_{q} A_{n(q)} .
$$

Then

$$
\tilde{\omega}_{n}\left(Q^{*} g_{t}(Q)\right)=\sum_{q, r} \omega_{n}\left(A_{q(n)}^{*} \alpha_{n}(t)\left(A_{r(n)}\right)\right)=\omega_{n}\left(A_{(n)}^{*} \alpha_{n}(t)\left(A_{(n)}\right)\right) .
$$

Hence

$$
\begin{aligned}
-\left.i(d / d t) \tilde{\boldsymbol{\omega}}_{n}\left(Q^{*} g_{t}(Q)\right)\right|_{t=0} & =\omega_{n}\left(A_{(n)}^{*}\left[H_{n}, A_{(n)}\right]\right) \\
& =\omega_{n}\left(A_{(n)}^{*} H_{n} A_{(n)}\right)-\omega_{n}\left(A_{(n)}^{*} A_{(n)} H_{n}\right) \\
& =\omega_{n}\left(A_{(n)}^{*} H_{n} A_{(n)}\right) \geqq 0,
\end{aligned}
$$

since $X \rightarrow \omega_{n}\left(A_{(n)}^{*} X A_{(n)}\right)$ is a positive linear functional and $H_{n} \geqq 0$ [20].

We claim that $\mathscr{D}_{0}=\pi\left(\mathfrak{B}\left(\mathfrak{H}_{0}\right)\right) \xi$ is a core for $H$, in fact for $H^{n}$ for all $n=1,2, \cdots$. To see this, we must verify

(i) that $\mathscr{D}_{0}$ is dense in $\mathscr{K}$,

(ii) that $\mathscr{D}_{0}$ is contained in the domain of $H$, and

(iii) that $\mathscr{D}_{0}$ is invariant under $U(t)=e^{i t H}$.

The claim then follows from the Singer-Poulsen-Segal core theorem [12, 22].

Assertion (i) has been verified in the proof of Lemmas 2 and 3 , 
specifically the estimates (26). Assertion (ii) is implied in the above computations, but the explicit argument has been omitted so we give some details below. Since $\mathfrak{A}_{0} \subset D(\delta)$, the vectors in $\pi\left(\mathfrak{A}_{0}\right) \xi$ are contained in $D(H)$ by (14) of Lemma 3. We show now that every element $\pi(Q) \xi$ with $Q=\left(A_{1}, t_{1}\right) \cdots\left(A_{k}, t_{k}\right)$ is in $D(H)$ if $A_{1}, \cdots, A_{k} \in \mathfrak{A}_{0}$. This is true since

$$
\pi(Q) \xi=U\left(t_{1}\right) \pi_{0}\left(A_{1}\right) U\left(t_{2}-t_{1}\right) \cdots U\left(t_{k}-t_{k-1}\right) \pi_{0}\left(A_{k}\right) \xi
$$

and both of the transformations $U(t)$ and $\pi_{0}(A)$ map $D(H)$ into itself for all $t \in \boldsymbol{R}$ and $A \in \mathfrak{A}_{0}$ as is shown in the Appendix. (More generally, if $f \in D(H)$ and $A \in D(\delta)$, then $\pi_{0}(A) f \in D(H)$ and

$$
H \pi_{0}(A) f=-i \pi_{0}(\delta(A)) f+\pi_{0}(A) H f,
$$

see the Appendix for details.)

Assertion (iii) is immediate from the formula

$$
U(t) \pi\left(\left(A_{1}, t_{1}\right) \cdots\left(A_{k}, t_{k}\right)\right) \xi=\pi\left(\left(A_{1}, t_{1}+t\right) \cdots\left(A_{k}, t_{k}+t\right)\right) \xi .
$$

(Since (i) through (iii) hold in the general setting of Lemma 3 , it is true in fact that $\pi\left(\mathfrak{B}\left(\mathfrak{H}_{0}\right)\right) \xi$ is a core for $H$ when $H$ is given by Lemma 3.)

Since $\pi\left(\mathfrak{B}\left(\mathfrak{A}_{0}\right)\right) \xi$ is a core for $H$, the conclusion $H \geqq 0$ follows from (34).

If $A_{1}, \cdots, A_{k}$ are arbitrary elements is $\mathfrak{A}$, then we have

$$
\tilde{\omega}\left(\left(A_{1}, t_{1}\right) \cdots\left(A_{k}, t_{k}\right)\right)=\left(\xi, \pi_{0}\left(A_{1}\right) U\left(t_{2}-t_{1}\right) \cdots U\left(t_{k}-t_{k-1}\right) \pi_{0}\left(A_{k}\right) \xi\right)
$$

for all $\left(t_{1}, \cdots, t_{k}\right) \in \boldsymbol{R}^{k}$. Let $E(d x)$ denote the spectral decomposition corresponding to $U(t)$ (Stone's theorem). Then $U(t)=\int_{0}^{\infty} e^{i t x} E(d x)$ and

$$
\tilde{\omega}\left(\left(A_{1}, t_{1}\right) \cdots\left(\mathrm{A}_{k}, t_{k}\right)\right)=\int e^{i\left(\left(t_{2}-t_{1}\right) x_{1}+\cdots+\left\langle t_{k}-t_{k-1}\right) x_{k-1}\right)} \mu\left(d x_{1} d x_{2} \cdots d x_{k-1}\right),
$$

where $\left.\mu\left(d x_{1} \cdots d x_{k-1}\right)=\left(\xi, \pi_{0}\left(A_{1}\right) E\left(d x_{1}\right)\right) \cdots E\left(d x_{k-1}\right) \pi_{0}\left(A_{k}\right) \xi\right)$, and where the integration is over $\prod_{j=1}^{k-1}\left\{x_{j}: x_{j} \geqq 0\right\}$. If we now define

$$
F\left(z_{1}, \cdots, z_{k}\right)=\int e^{\left.i\left(\mid t_{2}-z_{1}\right) x_{1}+\cdots+\left(z_{k}-z_{k-1}\right) x_{k-1}\right\}} \mu\left(d x_{1} \cdots d x_{k-1}\right),
$$

then it can be seen by induction that $F$ is analytic in $\operatorname{Im} z_{1}<\operatorname{Im} z_{2}<$ $\cdots<\operatorname{Im} z_{k}$ and has the desired boundary values.

In other words the condition is that there is an analytic function $R\left(\zeta_{1}, \cdots, \zeta_{k-1}\right)$ in $k-1$ variables on the product domain $\left\{\operatorname{Im} \zeta_{j}>0\right\}$ such that $R\left(t_{2}-t_{1}, \cdots, t_{k}-t_{k-1}\right)=\tilde{\omega}\left(\left(A_{1}, t_{1}\right) \cdots\left(A_{k}, t_{k}\right)\right)$.

The following lemma yields this conclusion.

Lemma 4. Let $H$ be a non-negative (generally unbounded) operator 
in a Hilbert space $\mathscr{K}$. Let $A_{1}, \cdots, A_{k+1}$ be a set of bounded operators on $\mathscr{K}$. Then the operator valued function

is

$$
A\left(z_{1}, \cdots, z_{k}\right)=A_{1} e^{i z_{1} H} A_{2} \cdots e^{i z_{k} H} A_{k+1}
$$

1) analytic on $V_{+}=\Pi\left\{\operatorname{Im} z_{j}>0\right\}$,

2) strongly continuous from $\bar{V}_{+}$to $B(\mathscr{K})$, and

3) uniformly bounded in operator norm,

$$
\left\|A\left(z_{1}, \cdots, z_{k}\right)\right\| \leqq \Pi\left\|A_{j}\right\| \text { for all }\left(z_{1}, \cdots, z_{k}\right) \in \bar{V}_{+} .
$$

Proof. If $E$ denotes the resolution of the identity for $H$, then by the spectral theorem,

$$
\begin{aligned}
\left\|e^{i z H} f\right\|^{2} & =\int_{0}^{\infty}\left|e^{i z x}\right|^{2}\|E(d x) f\|^{2}=\int_{0}^{\infty} e^{-2(\operatorname{Im} z) x}\|E(d x) f\|^{2} \\
& \leqq \int_{0}^{\infty}\|E(d x) f\|^{2} \leqq\|f\|^{2} \text { for all } z \text { in }\{\operatorname{Im} z \geqq 0\} .
\end{aligned}
$$

Hence, $e^{i z H}$ is a holomorphic family of contractions in $\operatorname{Im} z>0$, which is strongly continuous up to the boundary $\operatorname{Im} z=0$.

It is known [9] that an operator valued function, $A(z)$ say, is analytic if and only if the scalar functions $z \rightarrow(f, A(z) g)$ are analytic for all $f, g \in \mathscr{K}$. The conclusions of the lemma follow from this for the case $k=1$, since then $(f, A(z) g)=\left(A_{1}^{*} f, e^{i z H} A_{2} g\right)$.

Suppose that the conclusions 1 through 3 are satisfied for $k-1$. We show that they are also satisfied for $k$. Let $A_{1}, \cdots, A_{k+1}$ be bounded operators on $\mathscr{K}$, and let $f, g$ be vectors in $\mathscr{K}$. We show that $\left(f, A\left(z_{1}\right.\right.$, $\left.\left.\cdots, z_{k}\right) g\right)$ is analytic on $V_{+}(k)$. But

$$
\left(f, A\left(z_{1}, \cdots, z_{k}\right) g\right)=\left(e^{\tilde{i_{1} H}} A_{1}^{*} f, A^{\prime}\left(z_{2}, \cdots, z_{k}\right) g\right),
$$

where $A^{\prime}\left(z_{2}, \cdots, z_{k}\right)=A_{2} e^{i z_{2} H} \cdots e^{i z_{k} H} A_{k+1}$ satisfies conclusions 1 through 3 for $\left(z_{2}, \cdots, z_{k}\right)$ in $\bar{V}_{+}(k-1)$, and where $\widetilde{z}_{1}=-\operatorname{Re} z_{1}+i \operatorname{Im} z_{1}$. If $z_{1}$ is fixed, then the right hand side of (36) is analytic in the variables $z_{2}, \cdots, z_{k}$ by the induction hypothesis. If $z_{2}, \cdots, z_{k}$ are kept fixed, then it is analytic in the variable $z_{1}$ by the preceeding remark. Since $\operatorname{Im} z_{1}=\operatorname{Im} \widetilde{z}_{1}$, it follows by Hartogs' theorem [4] that $\left(f, A\left(z_{1}, \cdots, z_{k}\right) g\right)$ is analytic in $\left\{\operatorname{Im} z_{1}>0\right\} \times V_{+}(k-1)=V_{+}(k)$.

Quite similarly, we get conclusions 2 and 3 by induction. Estimation of the right hand side of (36) gives

$$
\begin{aligned}
\left|\left(f, A\left(z_{1}, \cdots, z_{k}\right) g\right)\right| & \leqq\left\|e^{i \tilde{z}_{1} H} A_{1}^{*} f\right\|\left\|A^{\prime}\left(z_{2}, \cdots, z_{k}\right) g\right\| \\
& \leqq\left\|A_{1}^{*} f\right\| \prod_{2}^{k}\left\|A_{j}\right\|\|g\| \leqq\left(\prod_{1}^{k}\left\|A_{j}\right\|\right)\|f\|\|g\| \text {. q.e.d. }
\end{aligned}
$$


There is an important corollary to the proof:

COROLLARY 3. Let $\delta$ be an inner limit derivation, and let $\left(\pi_{0}, \mathfrak{A}, \mathscr{K}, \xi, U(t)\right)$ be a dynamical system such that $\xi$ defines a ground state on $\mathfrak{B}(\mathfrak{H})$. Then the Hamiltonian $H=-\left.i(d / d t) U(t)\right|_{t=0}$ is nonnegative, and $\pi\left(\mathfrak{B}\left(\mathfrak{H}_{0}\right)\right) \xi$ is a core for $H$.

The conclusion $H \geqq 0$ is very important for several reasons. First of all, it shows that the Hamiltonian $H$ is physical (the energy is bounded below). The Friedrichs extension $H_{F^{\prime}}$ of $H_{0}$ shares this property with $H$. But the automorphism group implemented by $H_{F}$ cannot be used to describe the dynamics, because it does not leave the total algebra of observables invariant (cf. below). Of course, extensions of $H_{0}$ are not unique. Therefore the weak*-limit state $\omega$ alone does not determine a dynamics. The preceeding discussion shows that instead the state $\tilde{\omega}$ on $\mathfrak{B}(\mathfrak{A})$ leads to a useful dynamics. The constructions of both $H$ and $H_{F}$ are canonical (in terms of the given data.) The long established usefulness of $H_{F}$ undoubtedly derives from this fact (the canonical construction).

The conclusion that $\pi\left(\mathfrak{B}\left(\mathfrak{A}_{0}\right)\right) \xi$ is a core for $H$ is important, because it enables us to show that $H$ has non-negative spectrum. The conclusion $H \geqq 0$ in turn is in itself surprising in view of the fact that dilations of non-negative symmetric operators are not generally semibounded (let alone have non-negative spectrum), $[\mathrm{Ng}]$ and $[\mathrm{Ex}]$.

The following much weaker corollary is in fact contained in the statement of Theorem 5 .

CoROLlaRY 4. Let $\delta$ be an inner limit derivation in a $C^{*}$-algebra with unit. Then there exists a state $\omega_{0}$ on $\mathfrak{A}$ such that

$$
-i \omega_{0}\left(A^{*} \delta(A)\right) \geqq 0 \text { for all } A \in D(\delta) .
$$

It is important to note that the conclusion (37) is properly weaker than the conclusion $H \geqq 0$ of the previous corollary. To see this, we note that (37) can be established from the convergence of the one-time Green's functions alone, cf. (29).

The conclusion (37) implies that the form $-i \omega_{0}\left(A^{*} \delta(B)\right)$ determines a symmetric operator, $H_{0}$ say, in the Hilbert space $\mathscr{C}$ of the GNS representation corresponding to a given state $\omega_{0}$ satisfying (37). So in particular $H_{0} \geqq 0$. There is then an alternative way (the Friedrichs extension) of obtaing a self-adjoint and non-negative extension of $H_{0}$, even without extending the underlying Hilbert space $\mathscr{H}$.

Let $H_{F}$ denote the Friedrichs extension of $H_{0}$ and let $U_{F}(t)=e^{i t H_{F}}$ 
be the corresponding unitary group. Then, by Theorem 2, the state $\omega_{0}$ extends to a state $\omega_{F}$ on $\mathfrak{B}(\mathfrak{R})$, which is given by the formula (3) with $U(t)$ replaced by $U_{F}(t)$, and which is in fact a ground state.

An application of Theorem 1 to the state $\omega_{F}$ on $\mathfrak{B}(\mathfrak{A})$ leads to a unitary representation $\widetilde{U}_{F}(t)$ on the Hilbert space $\mathscr{K}_{\omega_{F}}$. But there is no reason to expect that the corresponding infinitesimal generator $-\left.i(d / d t) \widetilde{U}_{F}(t)\right|_{t=0}$ has a non-negative spectrum.

Moreover, the extensions $H$ constructed in Theorem 5 have several properties (in addition to $H \geqq 0$ ) that do not in general hold for the Friedrichs extensions $H_{F} \cdot{ }^{11}$

We list two:

Proposition 3. Let $\delta$ be an inner limit derivation in a $C^{*}$-algebra $\mathfrak{A}$ as above. Let $\left(\pi_{0}, \mathfrak{A}, \mathscr{K}, \xi, U(t)\right)$ be a dynamical system such that $\xi$ defines a ground state on $\mathfrak{B}(\mathfrak{H})$; and finally let $H$ denote the corresponding infinitesimal generator.

Then

(i ) The automorphism group $\bar{\alpha}_{t}(X)=U(t) X U(-t),(X \in B(\mathscr{K}), t \in \boldsymbol{R})$ leaves the von Neumann algebra $M=\pi(\mathfrak{B}(\mathfrak{H}))^{\prime \prime}$ invariant, i.e., $\bar{\alpha}_{t}(M)=M$ for all $t \in \boldsymbol{R}$.

(ii) There are two commuting non-negative self-adjoint operators $H^{\prime \prime}$ and $H^{\prime}$ such that $H^{\prime \prime}$ is affiliated with $M$ and $H^{\prime}$ with $M^{\prime}$, and $H=H^{\prime \prime}+H^{\prime}$.

Proof. The identity $U(t) \pi(Q) U(-t)=\pi\left(g_{t} Q\right)$ for $Q \in \mathfrak{B}(\mathfrak{A})$ and $t \in \boldsymbol{R}$ show that $\pi(\mathfrak{B}(\mathfrak{H}))$ is invariant under $\bar{\alpha}_{t}$. Since $\bar{\alpha}_{t}$ is continuous on $M$ with respect to the weak topology, the invariance of $M$ follows from the von Neumann-double commutant theorem [20]. So we have an automorphism group of $M$ which is implemented by a non-negative selfadjoint operator $H$. The conclusion (ii) follows from this and an application of Borcher's theorem [20].

5. Appendix (Extensions of derivations in representations.) The core property of Corollary 4 is satisfied for general Hamiltonians $H$ of dynamical systems which are generated by regular inner limit derivations in $C^{*}$-algebras. Explicitly:

TheORem A. Let $\delta$ be a regular inner limit derivation in a $\mathrm{C}^{*}$ algebra 2 . Let $\delta_{n}=\operatorname{ad} i H_{n}$ be a sequence of inner derivations which approximate $\delta$ on core $\mathfrak{A}_{0}$. Let $\left\{\omega_{n}\right\}$ be a corresponding sequence of

1) Problem: It is not known whether $H_{F}$ extends $\delta$ in the representation $\pi_{0}$. We do not know if the domain of $H_{F}$ is invariant under $\pi_{0}(A)$ for all $A \in D(\delta)$, or not. Cf. Appendix A. A special case of this problem is considered in [Ph, p. 382]. 
$\left(e^{i t H_{n}} \cdot e^{-i t H_{n}}\right)$-invariant states on $\mathfrak{A}$. Let $\tilde{\boldsymbol{\omega}}$ be a weak-*cluster state on $\mathfrak{B}(\mathfrak{A})$ of the sequence $\left\{\tilde{\omega}_{n}\right\}$ as in Lemma 2 and let $\left(\pi, \mathfrak{A}, \mathscr{K}, \xi, e^{i t H}\right)$ be the corresponding dynamical system.

Then $\pi\left(\mathfrak{B}\left(\mathfrak{A}_{0}\right)\right) \xi$ is a core for $H$; and

$$
\text { for all } A \in D(\delta) \text { and } f \in D(H) \text { we have } \pi(A) f \in D(H) \text { and }
$$

$$
i H \pi(A) f=\pi(\delta(A)) f+\pi(A) i H f .
$$

Proof. Put $\mathscr{D}_{0}=\pi\left(\mathfrak{B}\left(\mathfrak{H}_{0}\right)\right) \xi$ and $U(t)=e^{i t H}$. We have already noted that $\mathscr{D}_{0}$ is dense in $\mathscr{K}$ and invariant under $U(t)$. By the core theorem [12] it is then enough to show that $\mathscr{D}_{0}$ is contained in $D(H)$.

Elements in $\mathscr{D}_{0}$ are linear combinations of elements of the form $\pi\left(\left(B_{1}, t_{1}\right) \cdots\left(B_{l}, t_{l}\right)\right) \xi$ where $B_{i} \in \mathfrak{A}_{0}$. We show below that these elements are in the domain of $H$ and that

$$
i H \pi\left(\left(B_{1}, t_{1}\right) \cdots\left(B_{l}, t_{l}\right)\right) \xi=\sum_{i=1}^{l} \pi\left(\left(B_{1}, t_{1}\right) \cdots\left(\delta\left(B_{i}\right), t_{i}\right) \cdots\left(B_{l}, t_{l}\right)\right) \xi,
$$

or in a more compact notation

$$
i H \pi(Q) \xi=\pi(\tilde{\delta}(Q)) \xi,
$$

where $Q=\left(B_{1}, t_{1}\right) \cdots\left(B_{l}, t_{l}\right), \quad \tilde{\delta}(Q)=\sum_{i=1}^{l} Q_{i}$, and $Q_{i}=\left(B_{1}, t_{1}\right) \cdots\left(\delta\left(B_{i}\right)\right.$, $\left.t_{i}\right) \cdots\left(B_{l}, t_{l}\right)$. It is easy to verify that $\delta$ extends linearly to $\mathfrak{B}(D(\delta))$ as a derivation in $\mathfrak{B}(\mathfrak{R})$ this way. We denote the extension by $\tilde{\delta}$.

Suppose for the moment that the inclusion $\mathscr{D}_{0} \subset D(H)$ has been established. Then we show that (38) holds. Let $A \in \mathfrak{A}_{0}$ and $f \in D(H)$ be given. Since $\mathscr{D}_{0}$ is then a core by [12], there is a sequence of elements $Q_{n} \in \mathfrak{B}\left(\mathfrak{A}_{0}\right)$ such that $\pi\left(Q_{n}\right) \xi \rightarrow f$ and $i H \pi\left(Q_{n}\right) \xi=\pi\left(\tilde{\delta}\left(Q_{n}\right)\right) \xi \rightarrow i H f$, cf. (39) and (40). Consequently,

and

$$
\pi(A) \pi\left(Q_{n}\right) \xi \rightarrow \pi(A) f
$$

$$
\begin{aligned}
i H \pi(A) \pi\left(Q_{n}\right) \xi & =i H \pi\left(A Q_{n}\right) \xi=\pi\left(\tilde{\delta}\left(A Q_{n}\right)\right) \xi \\
& =\pi\left(\delta(A) Q_{n}\right) \xi+\pi\left(A \tilde{\delta}\left(Q_{n}\right)\right) \xi \\
& =\pi(\delta(A)) \pi\left(Q_{n}\right) \xi+\pi(A) \pi\left(\tilde{\delta}\left(Q_{n}\right)\right) \xi \\
& \rightarrow \pi(\delta(A)) f+\pi(A) i H f .
\end{aligned}
$$

Since $H$ is closed, the vector $\pi(A) f$ is in the domain of $H$ and the identity in (38) holds.

If $A \in D(\delta)$ is given, then there is a sequence of elements $A_{n} \in \mathfrak{A}_{0}$ such that $A_{n} \rightarrow A$ and $\delta\left(A_{n}\right) \rightarrow \delta(A)$. But then

and

$$
\pi\left(A_{n}\right) f \rightarrow \pi(A) f,
$$




$$
\begin{aligned}
i H \pi\left(A_{n}\right) f & =\pi\left(\delta\left(A_{n}\right)\right) f+\pi\left(A_{n}\right) i H f \\
& \rightarrow \pi(\delta(A)) f+\pi(A) i H f,
\end{aligned}
$$

so indeed $\pi(A) f \in D(H)$, and (38) holds also for $A \in D(\delta)$.

We now turn to the proof of the inclusion $\mathscr{D}_{0} \subset D(H)$. Let $P$ and $Q$ be elements in $\mathfrak{B}\left(\mathfrak{A}_{0}\right)$. We contend that equations (29), (30) and (31) hold with $A, B$ and $\delta$ replaced by $P, Q$ and $\tilde{\delta}$, respectively. To see this it is enough to verify (29), for then (30) and (31) follow as in the proof of Lemma 3. By linearity we may assume that $P=\left(A_{1}, s_{1}\right) \cdots\left(A_{k}, s_{k}\right)$ and $Q=\left(\left(B_{1}, t_{1}\right) \cdots\left(B_{l}, t_{l}\right)\right)$. By (25) and (24) there is a subsequence $\left\{n_{p}\right\}$ of the integers such that

$$
\begin{aligned}
\left.(d / d t) \dot{\omega}\left(P g_{t}(Q)\right)\right|_{t=0} & =\sum_{i=1}^{l}\left(\partial / \partial t_{i}\right) G\left(A_{1}, \cdots, A_{k}, B_{1}, \cdots B_{l} ; s_{1}, \cdots, s_{k}, t_{1} \cdots t_{l}\right) \\
& =\sum_{i=1}^{l} \lim _{n_{p}}\left(\partial / \partial t_{i}\right) \tilde{\omega}_{n_{p}}\left(\left(A_{1}, s_{1}\right) \cdots\left(B_{1}, t_{1}\right) \cdots\left(B_{l}, t_{l}\right)\right) \\
& =\sum_{i=1}^{l} \lim _{n_{p}} \tilde{\omega}_{n_{p}}\left(\left(A_{1}, s_{1}\right) \cdots\left(B_{1}, t_{1}\right) \cdots\left(\delta_{n_{p}}\left(B_{i}\right), t_{i}\right) \cdots\left(B_{l}, t_{l}\right)\right) \\
& =\sum_{i=1}^{l} \lim _{n_{p}} \tilde{\omega}_{n_{p}}\left(\left(A_{1}, s_{1}\right) \cdots\left(B_{1}, t_{1}\right) \cdots\left(\delta\left(B_{i}\right), t_{i}\right) \cdots\left(B_{l}, t_{l}\right)\right) \\
& =\sum_{i=1}^{l} \tilde{\omega}\left(P Q_{i}\right)=\tilde{\omega}(P \tilde{\delta}(Q)) \\
& =\left.(d / d t) \tilde{\omega}\left(g_{-1}(P) Q\right)\right|_{t=0}=-\tilde{\omega}(\tilde{\delta}(P) Q) .
\end{aligned}
$$

We used that

$$
\begin{aligned}
\mid \tilde{\omega}_{n_{p}} & \left(P\left(B_{1}, t_{1}\right) \cdots\left(\delta_{n_{p}}\left(B_{i}\right), t_{i}\right) \cdots\left(B_{l}, t_{l}\right)\right)-\tilde{\omega}_{n_{p}}\left(P Q_{i}\right) \mid \\
& =\left|\omega_{n_{p}}\left(\left(A_{1}, s_{1}\right) \cdots\left(B_{1}, t_{1}\right) \cdots\left(\delta_{n_{p}}\left(B_{i}\right)-\delta\left(B_{i}\right), t_{i}\right) \cdots\left(B_{l}, t_{l}\right)\right)\right| \\
& \leqq\left(\prod_{p=1}^{k}\left\|A_{p}\right\|\right)\left(\prod_{q \neq i}\left\|B_{q}\right\|\right)\left\|\delta_{n_{p}}\left(B_{i}\right)-\delta\left(B_{i}\right)\right\| \rightarrow 0,
\end{aligned}
$$

cf. (27).

Quite similarly, it follows from the regularity of $\delta$ that the Green's function $G\left(A_{1}, \cdots, A_{k}, B_{1}, \cdots, B_{l} ; s_{1}, \cdots, s_{k}, \cdots\right)$ is of class $C^{2}$ (cf. the proof of Lemma 3$)$, and that

$$
\left.\left(\frac{d}{d t}\right)^{2} \tilde{\boldsymbol{\omega}}\left(P g_{t}(Q)\right)\right|_{t=0}=-\tilde{\boldsymbol{\omega}}(\tilde{\delta}(P) \tilde{\delta}(Q)),
$$

so that we get

$$
\begin{aligned}
\|U(t) \pi(Q) \xi-\pi(Q) \xi-t \pi(\tilde{\delta}(Q)) \xi\|_{\mathscr{S}}^{2} \\
=2 \tilde{\omega}\left(Q^{*} Q\right)+t^{2} \tilde{\omega}\left(\tilde{\delta}\left(Q^{*}\right) \tilde{\delta}(Q)\right) \\
-\left[\tilde{\omega}\left(g_{t}\left(Q^{*}\right) Q\right)+\tilde{\omega}\left(Q^{*} g_{t}(Q)\right)\right]
\end{aligned}
$$




$$
\begin{aligned}
& -t\left[\tilde{\omega}\left(g_{t}\left(Q^{*}\right) \tilde{\delta}(Q)\right)+\tilde{\omega}\left(\tilde{\delta}\left(Q^{*}\right) g_{t}(Q)\right)\right] \\
& +t\left[\tilde{\omega}\left(Q^{*} \tilde{\delta}(Q)\right)+\tilde{\omega}\left(\tilde{\delta}\left(Q^{*}\right) Q\right)\right] \\
= & o\left(t^{2}\right)
\end{aligned}
$$

as in the proof of Lemma 3, now with $A$ replaced by $Q$. The desired conclusion $\pi(Q) \xi \in D(H)$ follows. $\quad$ q.e.d.

Acknowledgment. We are grateful to Professor S. Sakai for suggesting in a conversation the usefulness of possible infinitesimal characterizations of the KMS condition.

\section{REFERENCES}

[1] H. ARAKI, Wightman functions, retarded functions and their analytic continuations, Supp. Progress Theoretical Phys. 18 (1961), 83-125.

[2] H. ARAKI, Multiple time analyticity of a quantum statistical state satisfying the KMS boundary condition, Publ. RIMS, Kyoto Univ., Ser. A, 4 (1968), 361-371.

[3] H. ARAKI, Relative hamiltonian for faithful normal states of a von Newmann algebra, Publ. RIMS, Kyoto Univ. 9 (1973), 165-209.

[4] S. Bochner and W. T. Martin, Several Complex Variables, Princeton Univ. Press, Princeton, N. J. 1948.

[5] O. Bratteli and D. W. Robinson, Unbounded derivations of $C^{*}$-algebras, Commun. Math. Phys., 42 (1975), 253-268.

[6] O. Brattli, R. Herman and D. W. Robinson, Quasianalytic vectors and derivations of operator algebras, preprint, Bielefeld 1976.

[7] O. BRATteli aND D. W. RoBinson, Green's functions, hamiltonians and modular automorphisms, preprint, Bielefeld 1976.

[8] M. Duneau and B. Soutllard, Existence of Green's functions for dilute Bose gases, Commun. Math. Phys. 31 (1973), 113-125.

[9] E. Hille ANd R.S. Phillips, Functional Analysis and Semigroups, Amer. Math. Soc. Colloq. Publ. 31, Providence R. I. 1957.

[10] P. JerGenSEN, Trace states and KMS states for approximately inner dynamical one-parameter groups of *-automorphisms, Commun. Math. Phys. 53 (1977), 135-142.

[11] A. E. Nussbaum, Quasi-analytic vectors, Ark. Mat. 6 (1965) 179-191.

[12] N.S. Poulsen, On $C^{\infty}$-vectors and intertwining bilinear forms for representations of Lie groups, J. Functional Anal. 9 (1972), 87-120.

[13] R. T. Powers, Self-adjoint algebras of unbounded operators II, Trans. Amer. Math. Soc. 187 (1974), 261-293.

[14] R. T. Powers aNd S. SAKaI, Unbounded derivations in operator algebras, J. Functional Anal. 19 (1975), 81-95.

[15] R. T. Powers AND S. SAKAI, Existence of ground states and KMS states for approximately inner dynamics, Commun. Math. Phys. 39 (1975), 273-288.

[16] D. RUELLE, Some remarks on the ground state of infinite systems in statistical mechanics, Commun. Math. Phys. 11 (1969), 339-345.

[17] D. Ruelde, Analyticity of Green's functions of dilute quantum gases, J. Math. Phys. 12 (1971), 901-903.

[18] D. RuELle, Definition of Green's functions for dilute Fermi gases, Helv. Phys. Acta 45 (1972), 215-219. 
[19] M. B. RuskaI, Time development of quantum lattice systems, Commun. Math. Phys. 20 (1971), 193-204.

[20] S. SAKAI, $C^{*}$-algebras and $W^{*}$-algebras, Ergebnisse der Math. 60 N.Y., Springer-Verlag, 1971.

[21] I. E. SEgAL, Irreducible representations of operator algebras, Bull. Amer. Math. Soc. 53 (1947), 73-88.

[22] I. M. SINGER, Algebras of unbounded operators, Thesis, Univ. of Chicago, 1950.

[23] F. TRÈves, Topological Vector Spaces, Distributions and Kernels, Academic Press, N.Y. 1967.

[24] A.S. Wightman, Quantum field theoy in terms of vacuum expectation values, Phys. Rev. 101 (1956), 860-866.

[Ng] B. Sz.-NAGY, Extensions of linear transformations in Hilbert space which extend beyond this space, F. Ungar, New York 1960.

Added in proof. Additional references

[Ex] P. ExNER, Remark on the energy spectrum of a decaying system, Comm. Math. Phys. 50 (1976), 1-10.

[Ph] R. S. PHILliPs, The extension of dual subspaces invariant under an algebra, in Proc. of the International Symp. on Linear Spaces, Jerusalem 1960.

After the completion of this article we learned that KMS states of the algebra $\mathfrak{B}(\mathfrak{A})$ have been considered previously also in the article "Time translations in the algebraic formulation of statistical mechanics" (J. Math. Phys. 11 (1970), 2990-2998) by D. A. Dubin and G. L. Sewell. Although their Proposition 4 is similar to our Theorem 4, our approach is quite different, and in several respects more general. We are grateful to Professor Sewell for calling our attention to his work in this area.

DEPARTMENT OF MATHEMATICS

STANFORD UNIVERSITY

Stanford, California 94305

U. S. A. 
